\title{
Periostin promotes liver steatosis and hypertriglyceridemia through downregulation of PPAR $\alpha$
}

\author{
Yan Lu, ${ }^{1}$ Xing Liu, ${ }^{1}$ Yang Jiao, ${ }^{1}$ Xuelian Xiong, ${ }^{1}$ E Wang, ${ }^{1}$ Xiaolin Wang, ${ }^{1}$ Zhijian Zhang, ${ }^{1}$ Huijie Zhang, ${ }^{1}$ Lingling Pan, ${ }^{1}$ Youfei Cuan, ${ }^{2}$ \\ Dongsheng Cai, ${ }^{3}$ Guang Ning, ${ }^{1,4}$ and Xiaoying Li $i^{1,4,5}$ \\ 'Shanghai Institute of Endocrinology and Metabolism, Department of Endocrine and Metabolic Diseases, Shanghai Clinical Center for Endocrine and Metabolic Diseases, Ruijin Hospital, Shanghai Jiao \\ Tong University School of Medicine, Shanghai, China. ${ }^{2}$ Shenzhen University Diabetes Center, Shenzhen University Health Science Center, Shenzhen, China. ${ }^{3}$ Department of Molecular Pharmacology, Albert \\ Einstein College of Medicine, New York, New York, USA. ${ }^{4}$ The Key Laboratory of Endocrine Tumors and the Division of Endocrine and Metabolic Diseases, E-Institute of Shanghai Universities, Shanghai, China. \\ ${ }^{5}$ Chinese-French Laboratory of Cenomics and Life Sciences, Ruijin Hospital, Shanghai Jiao Tong University School of Medicine, Shanghai, China.
}

Hepatosteatosis is characterized by an aberrant accumulation of triglycerides in the liver; however, the factors that drive obesity-induced fatty liver remain largely unknown. Here, we demonstrated that the secreted cell adhesion protein periostin is markedly upregulated in livers of obese rodents and humans. Notably, overexpression of periostin in the livers of WT mice promoted hepatic steatosis and hypertriglyceridemia. Conversely, both genetic ablation of periostin and administration of a periostin-neutralizing antibody dramatically improved hepatosteatosis and hypertriglyceridemia in obese mice. Overexpression of periostin resulted in reduced expression of peroxisome proliferator-activated receptor $\alpha$ (PPAR $\alpha$ ), a master regulator of fatty acid oxidation, and activation of the JNK signaling pathway. In mouse primary hepatocytes, inhibition of $\alpha 6 \beta 4$ integrin prevented activation of JNK and suppression of PPAR $\alpha$ in response to periostin. Periostin-dependent activation of JNK resulted in activation of c-Jun, which prevented ROR $\alpha$ binding and transactional activation at the Ppara promoter. Together, these results identify a periostin-dependent pathway that mediates obesity-induced hepatosteatosis.

\section{Introduction}

Triglycerides (TGs) are usually stored in adipose tissue as an energy source. However, aberrant TG accumulation in peripheral tissues, such as the liver, is one aspect of the metabolic syndrome and is associated with the development of type 2 diabetes, atherosclerosis, hypertension, and even coronary heart disease (1-3).

In humans, obesity is tightly associated with an increased risk of nonalcoholic fatty liver disease (NAFLD) (4). Hepatosteatosis occurs when TG homeostasis is disrupted, due to increased de novo lipogenesis and fatty acid uptake and reduced fatty acid oxidation and VLDL export $(4,5)$. However, the molecular mechanisms of obesity-induced fatty liver remain largely unknown.

Periostin (encoded by Postn) is a secreted cell adhesion protein belonging to the fasciclin family (6). Initially, periostin was thought to function as a homophilic adhesion molecule during bone formation and support osteoblastic cell attachment through binding to $\alpha v \beta 3$, $\alpha v \beta 5$, or $\alpha 6 \beta 4$ integrins (7). Analysis of Postn-null mice has revealed that periostin participates in the development of bone and tooth formation, acting on mesenchymal cells (6). Recently, increasing evidence also suggests that periostin is involved in the development of multiple tumors via several signaling pathways, such as PI3K/AKT and $\mathrm{Wnt} / \beta$-catenin (8-11). In agreement with this, periostin expression is markedly upregulated in various human tumors, including in

Authorship note: Yan Lu, Xing Liu, and Yang Jiao contributed equally to this work Conflict of interest: The authors have declared that no conflict of interest exists. Submitted: November 25, 2013; Accepted: May 22, 2014.

Reference information: / Clin Invest. 2014;124(8):3501-3513. doi:10.1172/JCI74438. head and neck, breast, colon, pancreatic, and ovarian cancers $(7,12)$. However, the role of periostin in the regulation of TG homeostasis and in the pathogenesis of NAFLD remains uncharacterized.

In the present study, we identified a marked increase in periostin expression in the livers of obese mice and humans with fatty liver disease. Manipulation of periostin expression in the liver altered hepatic TG accumulation and serum TG levels.

\section{Results}

Hepatic periostin expression is upregulated in obese mice and humans with fatty liver disease. In order to identify genes that are potentially involved in dysfunctional hepatic lipid homeostasis in obesity, we previously performed a clustering analysis of Affymetrix arrays (13), which revealed that numerous mRNAs were markedly upregulated in the liver of mice fed a high-fat diet (HFD) compared with mice fed a normal chow diet (ND). In the liver of HFD-fed mice, expression levels of 7,198 genes were significantly changed $(P<0.05)$, of which 3,431 (47.7\%) were increased and 3,767 (52.3\%) were decreased. Our data showed a pronounced overexpression of periostin in the liver of HFD mice (Supplemental Table 1; supplemental material available online with this article; doi:10.1172/JCI74438DS1). Compared with that in primary nonhepatocytes isolated from control mice, periostin expression was 8-fold higher in mouse primary hepatocytes (MPHs; Supplemental Figure 1A), which indicates that hepatic parenchymal cells represent the main source of periostin expression. Increased Postn mRNA and periostin protein expression in HFD-fed mice was further confirmed by quantitative realtime RT-PCR (qRT-PCR) and ELISA, respectively (Figure 1A). To 

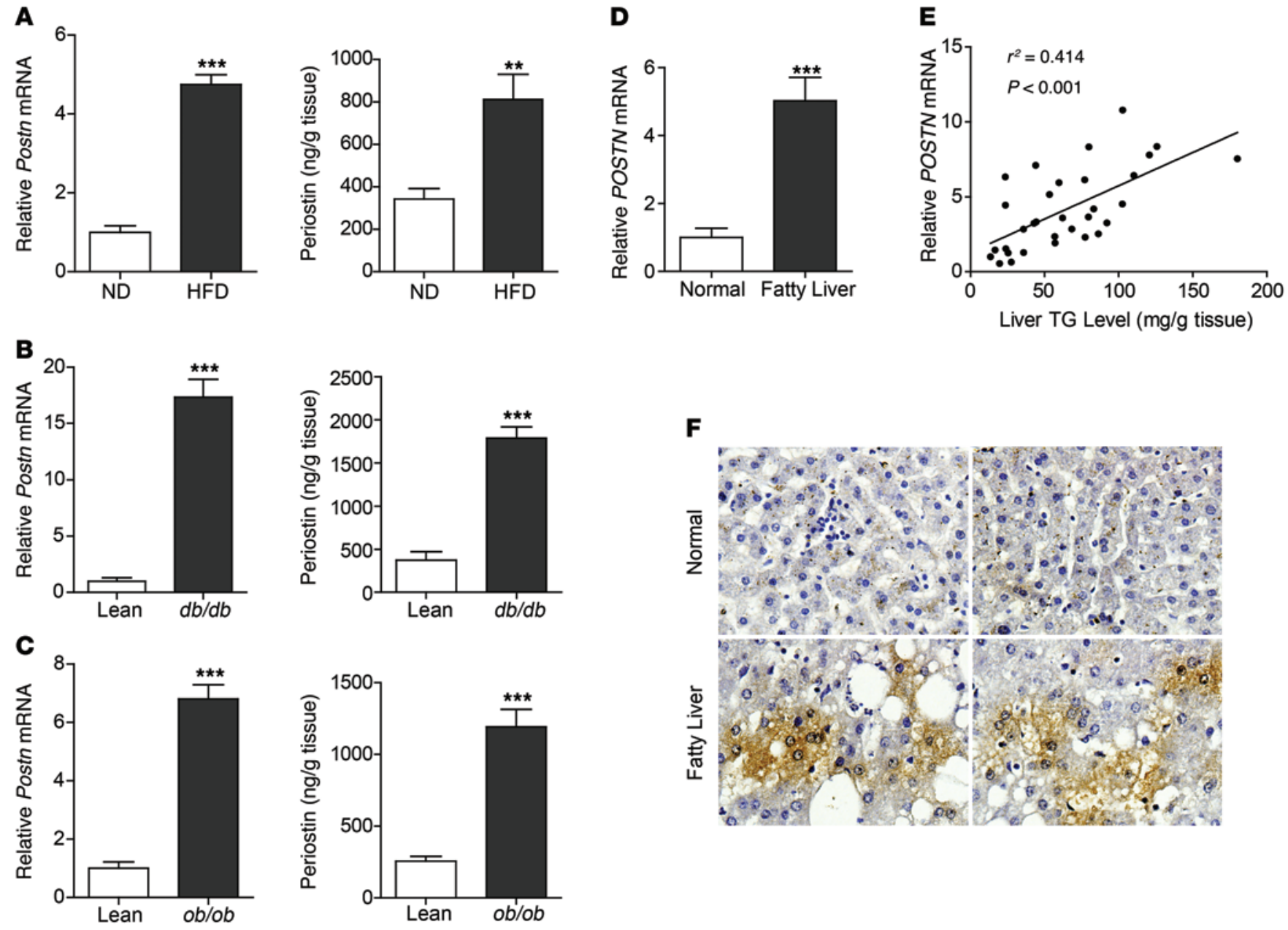

Figure 1. Hepatic expression of periostin is increased in fatty livers. (A) Hepatic Postn mRNA and periostin protein levels, determined by qRT-PCR and ELISA, in C57BL/6 mice. 8-week-old mice were fed ND or HFD for 12 weeks $(n=6)$. (B and C) Hepatic Postn mRNA and periostin protein levels, determined by qRT-PCR and ELISA, in $d b / d b$ (B) and ob/ob (C) mice $(n=6-9)$. (D) Relative mRNA expression of POSTN in livers from NAFLD patients $(n=17)$ and normal subjects $(n=15)$, determined by qRT-PCR. (E) Pearson $R$ and $P$ value for normalized POSTN mRNA levels versus TC content in human livers $(n=32)$. (F) Representative immunohistochemistry staining of periostin in liver sections from 2 normal subjects and NAFLD patients. Original magnification, $\times 200$. ${ }^{* *} P<0.01,{ }^{* * *} P<0.001$.

test whether the upregulation of periostin in the liver represents a more general feature of obesity-related hepatosteatosis, we examined periostin expression in $o b / o b$ and $d b / d b$ mice as independent standard models for monogenic-induced obesity (14). Indeed, Postn mRNA and periostin protein levels were markedly increased in the liver of the obese mice compared with lean controls (Figure 1, B and C). Interestingly, serum periostin levels were also elevated in obese mice (Supplemental Figure 1, B-D). Blood glucose levels and insulin sensitivity in HFD, $o b / o b$, and $d b / d b$ mice are also shown in Supplemental Figure 1, E-G. Additionally, an increase of hepatic Postn mRNA was observed in HFD rats (Supplemental Figure 1H).

Importantly, hepatic POSTN levels were dramatically increased in NAFLD patients and correlated well with hepatic TG content (Figure 1, D and E). The upregulation of hepatic POSTN in NAFLD patients was also confirmed by immunohistochemistry staining (Figure 1F). Moreover, serum periostin levels were also increased in human NAFLD patients (Supplemental Figure 1I, Supplemental Table 2, and ref. 15), although a significant correlation between serum periostin levels and hepatic TG content was not observed (data not shown). Therefore, our results substantiate the notion that the overproduction of periostin in the liver highlights an unanticipated and conserved feature of hepatosteatosis in obese rodents and humans.

Hepatic periostin expression is regulated by glucose. To clarify the physiological regulation of periostin in the liver, we investigated the effects of nutrients on periostin expression in vivo and in vitro. We used a continuous glucose infusion model as previously described (16). Infusion with $50 \%$ glucose $(2 \mathrm{ml} / \mathrm{h})$ via the jugular vein for 24 and 48 hours caused a sustained and significant increase in periostin expression in the liver of rats (Supplemental Figure 2A). Moreover, periostin levels were greater in the livers of C57BL/6 mice upon refeeding than in those fasted for 24 hours (Supplemental Figure 2B). We also examined Postn mRNA expression in cultured HepG2 or MPHs; addition of glucose at 15 and $25 \mathrm{mM}$ dramatically increased Postn expression in a dose-dependent manner, whereas neither insulin, the cAMP agonist forskolin, nor the glucocorticoid analog dexamethasone changed Postn expression in these cells (Supplemental Figure 2, C and D).

Recent studies reported that the transcription factor carbohydrate responsive element-binding protein (ChREBP) plays a 
A

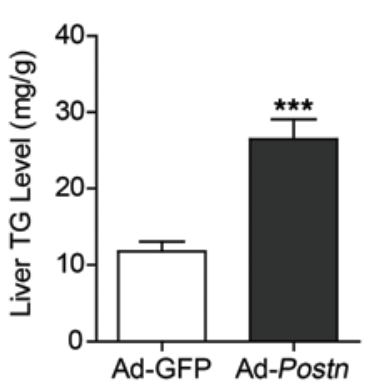

B

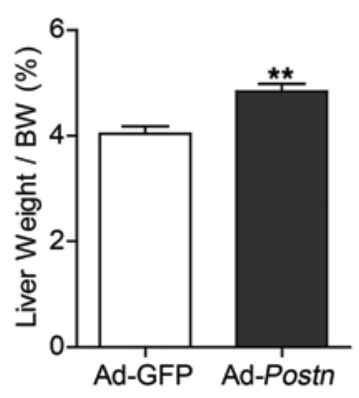

C

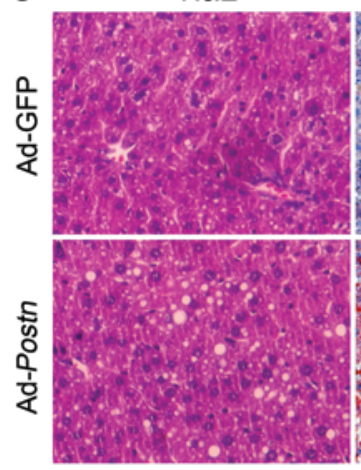

Oil Red O

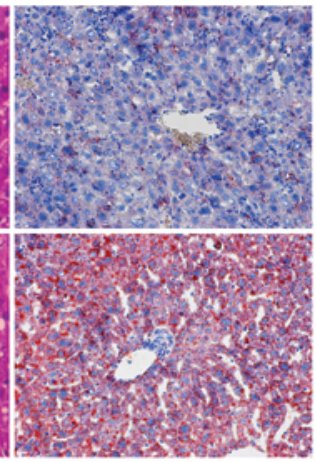

D

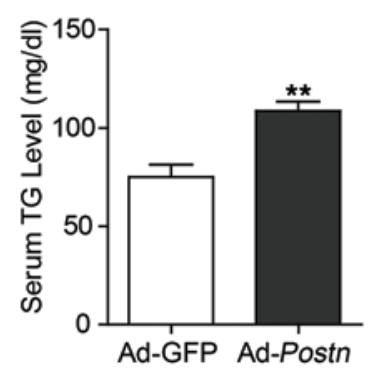

E

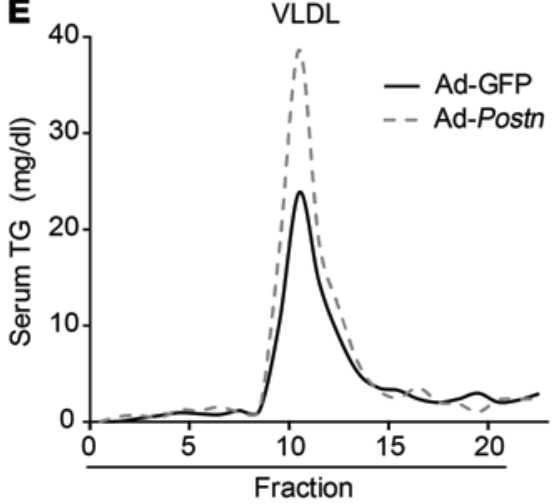

$\mathbf{F}$

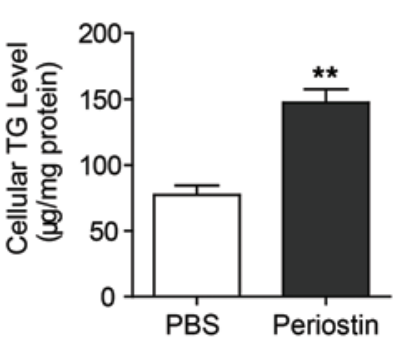

G

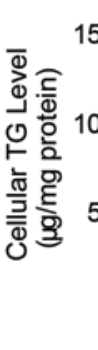

$\mathrm{MPH}$

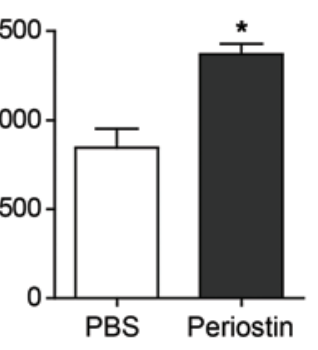

Figure 2. Periostin promotes hepatic TG accumulation in vivo and in vitro. (A and B) Liver TC content (A) and weight (B) in C57BL/6 mice infected with adenovirus (Ad-) containing GFP or Postn by means of tail vein injection at day 0 . After an overnight fast, mice were sacrificed at day 10 . Serum and liver tissues were collected for further analysis ( $n=6-7)$. (C) Representative histology (H\&E, left) or Oil Red 0 (right) staining showing TC accumulation in livers from mice injected with Postn versus GFP adenovirus. Original magnification, $\times 200$. (D and E) Serum TC (D) and lipoprotein-associated TC (E) in mice transduced with GFP or Postn adenovirus. (F and G) Cellular TC content in HepC2 cells (F) and MPHs (G). Cells were treated with recombinant periostin protein $(50 \mathrm{ng} / \mathrm{ml})$ or PBS vehicle control for 36 hours. ${ }^{*} P<0.05,{ }^{*} P<0.01,{ }^{* *} P<0.001$.

critical role in the induction of glucose-regulated genes in the liver $(17,18)$. Therefore, we used shRNA adenovirus to silence ChREBP in HepG2 cells, which resulted in a reduced response of periostin to high glucose (Supplemental Figure 3, A and B). We further generated a luciferase reporter construct containing the region of $-1,037 \mathrm{bp}$ to $+10 \mathrm{bp}$ of the mouse Postn promoter. The luciferase assay showed that the transcriptional activity containing this region was dramatically upregulated by ChREBP (Supplemental Figure 3C). By serial deletion within this region, we were able to define a potential ChREBP-binding site, located -356 bp to $-340 \mathrm{bp}$ upstream of the transcriptional start site (Supplemental Figure 3C). Moreover, mutation of this site largely abolished the regulation of ChREBP in periostin transcriptional activity (Supplemental Figure 3D), which indicates that this binding site is required for the induction of promoter activities by ChREBP. In addition, ChIP assays also showed that ChREBP was able to bind to this region of the Postn promoter in HepG2 cells exposed to high glucose (Supplemental Figure $3 \mathrm{E})$. Thus, multiple lines of evidence suggest that increased periostin expression in the liver is due, at least in part, to overnutrition.

Periostin promotes TG accumulation in the liver. To elucidate the pathophysiological role of periostin in the liver, we overexpressed periostin in the liver of C57BL/6 mice by delivering an adenoviral vector with the Postn gene via tail vein injection (Supplemental Figure 4A). At day 10 after injection, overexpression of periostin resulted in a significant increase in TG content and liver weight (Fig- ure 2, A and B). The prominent hepatosteatosis was also confirmed by $\mathrm{H} \& \mathrm{E}$ and Oil Red $\mathrm{O}$ staining (Figure 2C). In parallel, serum TG as well as serum VLDL fractionation was elevated (Figure 2, $\mathrm{D}$ and $\mathrm{E})$. To determine the rate of VLDL secretion, we injected tyloxapol, an inhibitor of plasma lipases, into mice overexpressing periostin or GFP. Hepatic VLDL secretion was unaffected by periostin overexpression (Supplemental Figure 4B). Moreover, there was no significant change in BW, serum and hepatic cholesterol levels, or liver damage markers in periostin-overexpressing mice (Supplemental Figure 4, C-G).

We further treated HepG2 cells with $50 \mathrm{ng} / \mathrm{ml}$ recombinant periostin protein and found that the cellular TG content was dramatically increased (Figure $2 \mathrm{~F}$ and Supplemental Figure 5A). Similar results were also obtained in MPHs (Figure $2 \mathrm{G}$ ). The dose of periostin was chosen based on cell proliferation assays (Supplemental Figure 5B) and previous reports (8). Collectively, our results indicate that periostin promotes TG accumulation in the liver.

Periostin promotes hepatic TG accumulation through downregulation of PPAR $\alpha$. We sought to investigate the molecular basis for periostin's promotion of hepatic TG accumulation. Hepatic TG storage is caused by an imbalance of fatty acid oxidation and de novo lipogenesis $(5,19)$. Mice overexpressing periostin showed reduced serum $\beta$-hydroxybutyrate levels (Figure $3 \mathrm{~A}$ ), indicative of decreased hepatic fatty acid oxidation. PPAR $\alpha$ (encoded by Ppara), a master regulator of mitochondrial and peroxisomal fatty acid oxidation 
A

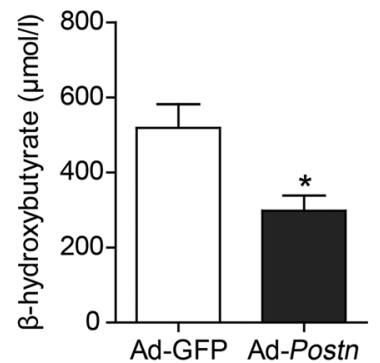

E

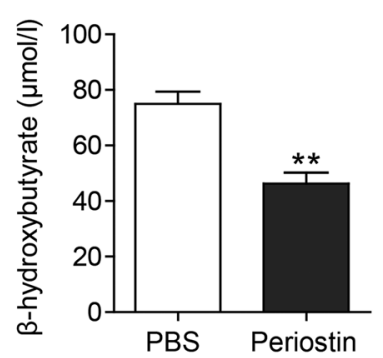

H

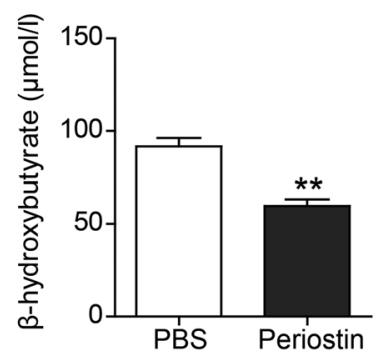

K
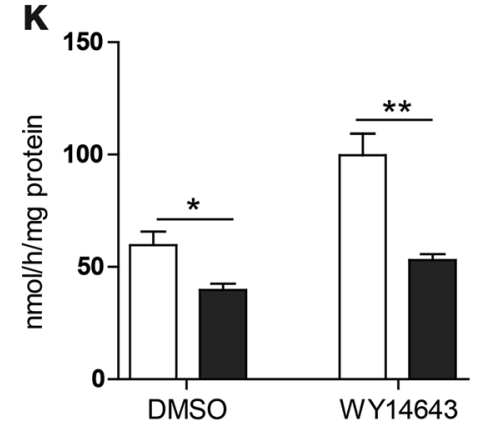

M $\square$ PBS $\square$ Periostin

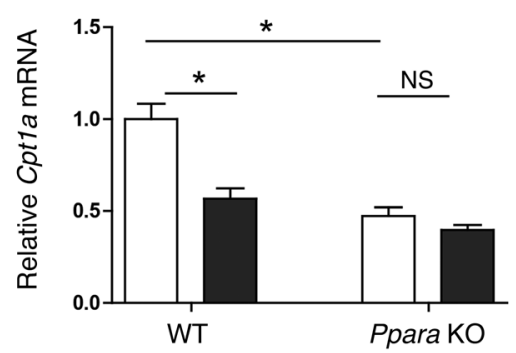

C

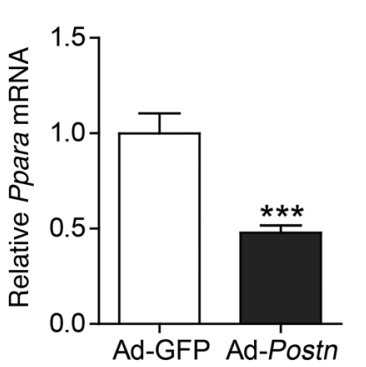

$\mathbf{F}$

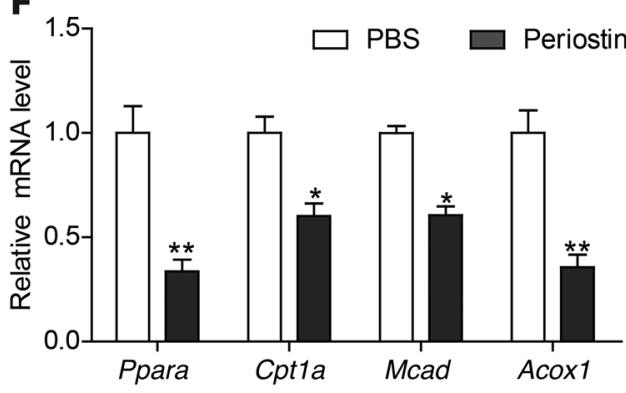

I

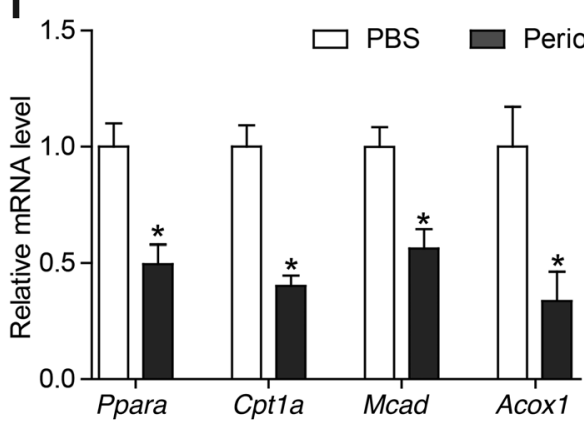

$\mathbf{L}$
D

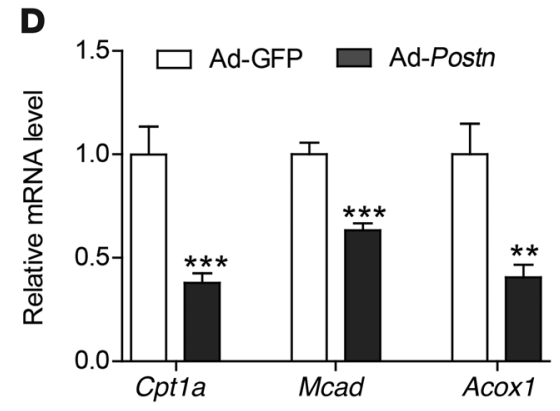

G

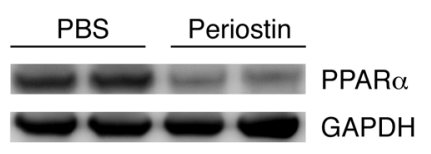

J
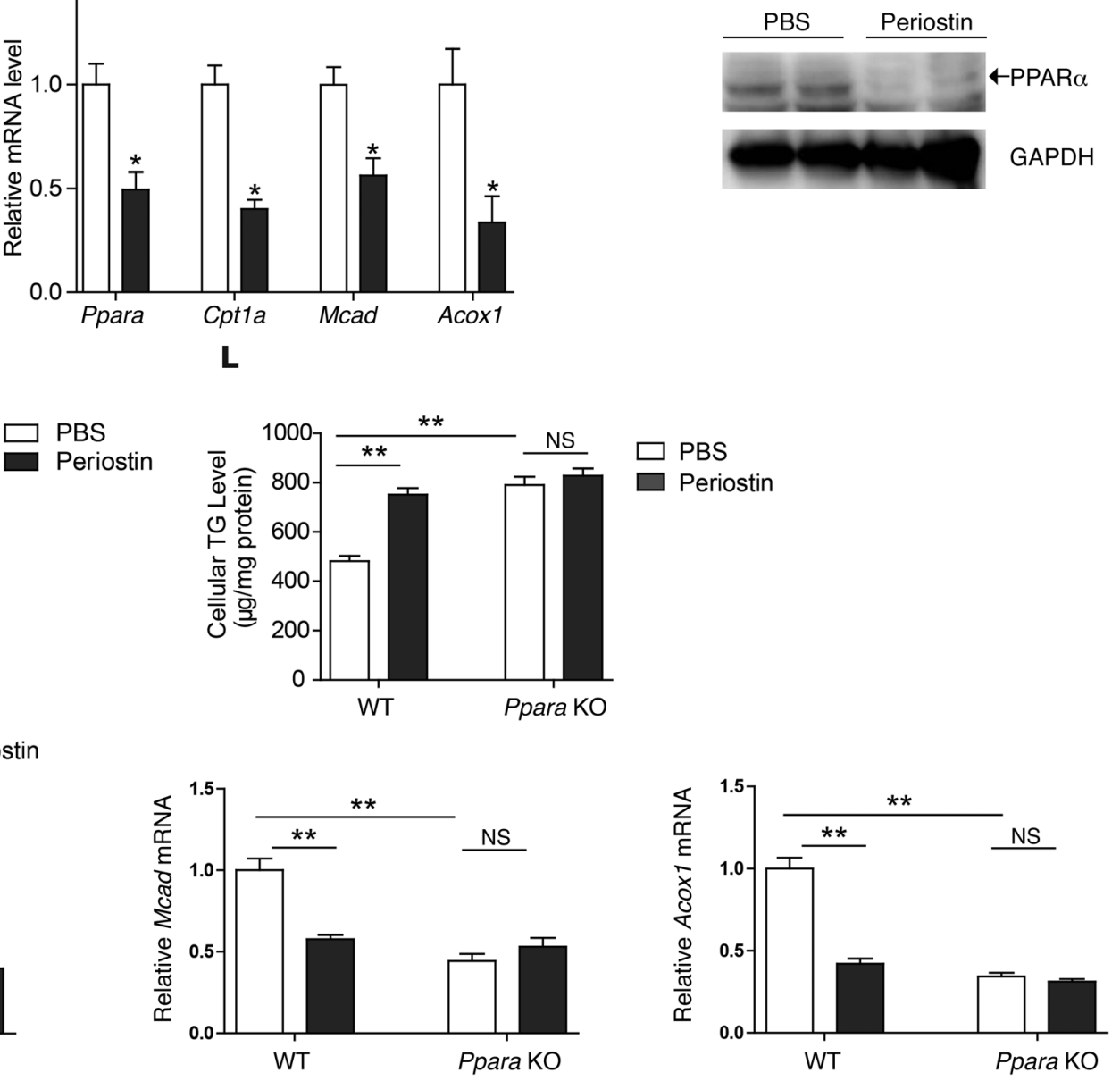
Figure 3. Periostin regulates liver lipid metabolism via PPARa. (A) Serum $\beta$-hydroxybutyrate levels in mice transduced with GFP or Postn adenoviruses $(n=6-7)$. (B and $\mathbf{C})$ mRNA expression of hepatic Ppara (B) and protein expression of hepatic PPAR $\alpha$ (C) in mice. (D) mRNA levels of hepatic Cpt1a, Mcad, and Acox1 in mice. (E) $\beta$-hydroxybutyrate levels in the medium of HepG2 cells. Cells were treated with periostin protein $(50 \mathrm{ng} / \mathrm{ml})$ or PBS vehicle control for 36 hours. (F) mRNA levels of Ppara, Cpt1a, Mcad, and Acox1 in HepG2 cells as in E. (C) Representative PPAR $\alpha$ protein levels in HepG2 cells. (H) $\beta$-hydroxybutyrate levels in the medium of MPHs. Cells were treated with periostin protein $(50 \mathrm{ng} / \mathrm{ml})$ or PBS for 36 hours. (I) mRNA levels of Ppara, Cpt1a, Mcad, and Acox1 in MPHs as in H. (J) Representative PPAR $\alpha$ protein levels in MPHs. (K) ${ }^{3} \mathrm{H}$-palmitate oxidation rate in MPHs treated with periostin protein or PBS. MPHs were preincubated in maintenance medium for 24 hours in the presence of WY14643 $(20 \mu \mathrm{M})$ or DMSO followed by incubation with $125 \mathrm{mM}^{3} \mathrm{H}$ palmitic acid and $1 \mathrm{mM}$ carnitine in PBS for 2 hours. ${ }^{3} \mathrm{H}_{2} \mathrm{O}$ was then measured. $n=4$. (L) Cellular TC content in MPHs isolated from WT or Ppara KO mice. Cells were treated with recombinant periostin protein (50 ng/ml) or PBS for 36 hours $(n=4-5)$. (M) mRNA levels of Cpt1a, Mcad, and Acox1 in MPHs. ${ }^{*} P<0.05,{ }^{* *} P<0.01,{ }^{* * *} P<0.001$.

(20,21), was particularly reduced (Figure 3, B and C). The MPHs isolated from Ppara KO mice were used as positive and negative controls for PPAR $\alpha$ Ab (Supplemental Figure 6A). Expression levels of the PPAR $\alpha$ target genes carnitine palmitoyltransferase 1A (Cpt1a), medium-chain acylcoenzyme-A dehydrogenase (Mcad), and acylCoA oxidase 1, palmitoyl (Acox1), were also downregulated (Figure 3D), whereas genes involved in lipogenesis and lipid transport remained unaffected (Supplemental Figure 6B). In cultured HepG2 cells and MPHs, periostin treatment also led to decreased $\beta$-hydroxybutyrate in the culture medium and reduced expression of fatty acid oxidation-related genes (Figure 3, E-J, and Supplemental Figure 6, C and D). In addition, $\beta$-oxidation of ${ }^{3} \mathrm{H}$-palmitate was significantly lower in MPHs treated with periostin than the vehicle control, even in the presence of the PPAR $\alpha$ agonist WY14643 (Figure 3K). PPAR $\alpha$ target genes were also downregulated by periostin in MPHs in the presence of WY14643 (Supplemental Figure 6E).

Next, we used MPHs isolated from Ppara KO mice. As expected, Ppara KO MPHs displayed an increase of TG content (Figure 3L). Notably, periostin increased cellular TG levels only in WT, not in Ppara KO, MPHs (Figure 3L). Consistent with this result, periostin reduced fatty acid oxidation-related genes only in WT MPHs (Figure $3 \mathrm{M}$ ), underlying the role of periostin in suppressing PPAR $\alpha$ function in this context.

Periostin downregulates PPAR $\alpha$ through $\alpha 6 \beta 4$ integrin-mediated JNK activation. To explore the molecular mechanism by which periostin downregulates PPAR $\alpha$ expression, we examined potential regulatory pathways. We found that periostin overexpression resulted in marked activation of the JNK signaling pathway, as JNK phosphorylation was increased, while the p38, ERK, and AKT pathways were unaffected (Figure 4A). In addition, phosphorylation of c-Jun, a downstream target of JNK kinase, was also enhanced by periostin overexpression (Figure 4A). The JNK pathway was also activated in HepG2 cells and MPHs treated with periostin (Figure 4, B and C).

Recent studies demonstrate that periostin can bind to the $\alpha v \beta 3$, $\alpha v \beta 5$, or $\alpha 6 \beta 4$ integrins (7). Our RT-PCR analysis showed that these integrins were expressed in the liver of C57BL/6 mice (Supplemental Figure 7A). To probe which of these integrins mediates periostin action, we preincubated MPHs with Abs against each pair of these integrins and measured the levels of phosphorylated
JNK induced by periostin. Periostin activated the JNK pathway primarily through the $\alpha 6 \beta 4$ integrins, while the Abs against the $\alpha v \beta 3$ and $\alpha v \beta 5$ integrins did not block JNK phosphorylation (Figure 4D). Consistently, the Abs against the $\alpha 6 \beta 4$ integrins also attenuated the cellular TG content and restored periostin-suppressed Ppara expression levels (Figure 4, E and F).

To further investigate the role of JNK kinase activation in hepatosteatosis, we used 2 approaches to block JNK function and then examined the effect of periostin. First, mice overexpressing periostin by adenoviral Post $n$ were treated with SP600125, a classic JNK kinase inhibitor (22). SP600125 abrogated the effect of periostin on liver weight and TG content in C57BL/6 mice (Supplemental Figure 7, B and C). The elevated serum TG and reduced serum $\beta$-hydroxybutyrate levels induced by periostin were also blocked by SP600125 (Supplemental Figure 7, D and E). Furthermore, the downregulation of PPAR $\alpha$ and its target genes was also abrogated by SP600125 treatment (Supplemental Figure 7, F and G).

Additionally, MPHs isolated from WT and Jnk1 KO mice were used. Periostin treatment led to a dramatic increase in cellular TG content and decrease in fatty acid oxidation-related genes in WT MPHs, but not Jnk1 KO MPHs (Figure 4, G-I). Taken together, these data firmly establish that periostin promotes hepatosteatosis by activating the JNK pathway via $\alpha 6 \beta 4$ integrins.

Previous studies have shown that several integrins could activate $\operatorname{Rac} 1(23,24)$, the upstream regulator of JNK signaling $(25,26)$. We explored whether periostin could activate Rac1 through $\alpha 6 \beta 4$ integrins. As evidenced by its translocation to the cell membrane, periostin definitely activated Rac1, which was attenuated by the Abs against $\alpha 6 \beta 4$ integrins (Supplemental Figure 8A). To further determine whether Rac1 is required for JNK activation by periostin, we pretreated HepG2 cells with the Rac1 antagonist NSC23766 (27), which largely abolished the JNK phosphorylation induced by periostin (Supplemental Figure 8B). Similar results were also obtained in HepG2 cells transfected with siRNA targeting Rac1 (Supplemental Figure 8C). Therefore, our results suggest that activation of JNK signaling by periostin and/or $\alpha 6 \beta 4$ integrins is dependent, at least in part, on Rac1.

To investigate the mechanisms of PPAR $\alpha$ downregulation by JNK signaling, we examined the expression of several upstream regulators of PPAR $\alpha$, including Hnf4, Coup-TFII, and Car (28-30), which were not altered by periostin (Supplemental Figure 9A). In accordance with the regulation of PPAR $\alpha$ expression, periostin inhibited Ppara promoter activity (Figure 5A). Using a series of truncated promoters, we defined a minimal periostin-responsive region (261 bp upstream of the transcriptional start site) that contained consensus binding sites for ATF/CREB, SP1, and ROR $\alpha$ (Figure 5, A and B). Site-directed mutagenesis showed that the ROR $\alpha$ binding site (located between $-57 \mathrm{bp}$ and $-40 \mathrm{bp}$ ) was indispensable for periostin inhibition of Ppara promoter activity (Figure 5, $\mathrm{B}$ and $\mathrm{C})$. Indeed, treatment of HepG2 cells and MPHs with cholesterol sulfate (CS), a ROR $\alpha$ agonist (31), increased PPAR $\alpha$ expression (Supplemental Figure 9B). Moreover, the luciferase activity of the Ppara promoter, which was dramatically enhanced by ROR $\alpha$ and CS, was largely abolished by mutation of this binding site (Supplemental Figure 9C). Therefore, these results suggest that ROR $\alpha$ may play a crucial role in mediating the periostin suppression of Ppara gene transcription. 

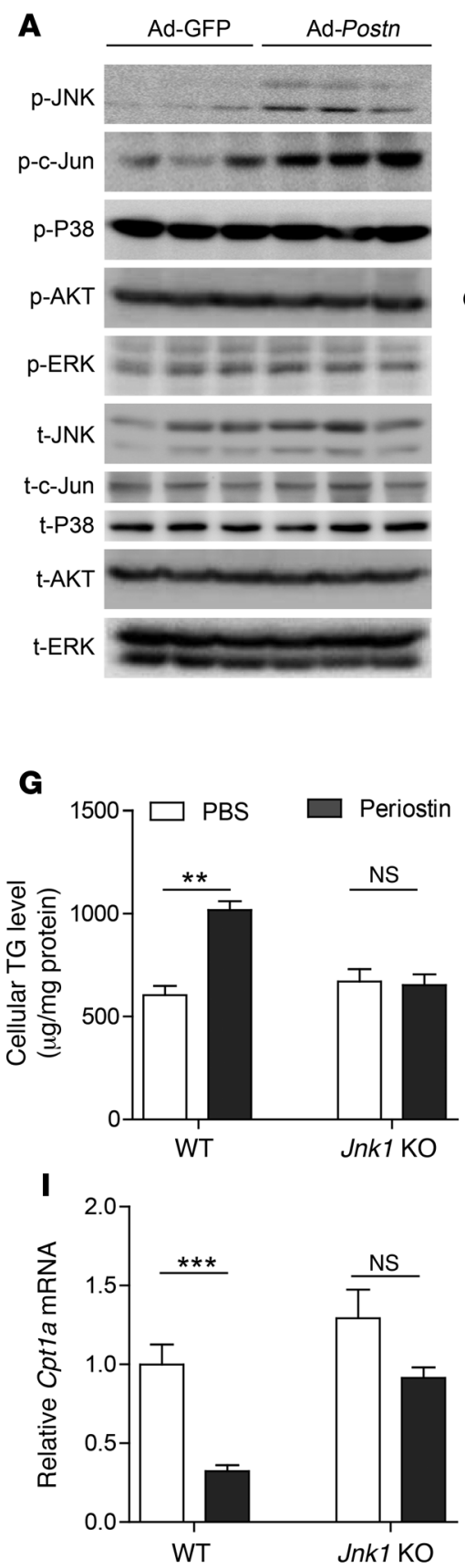
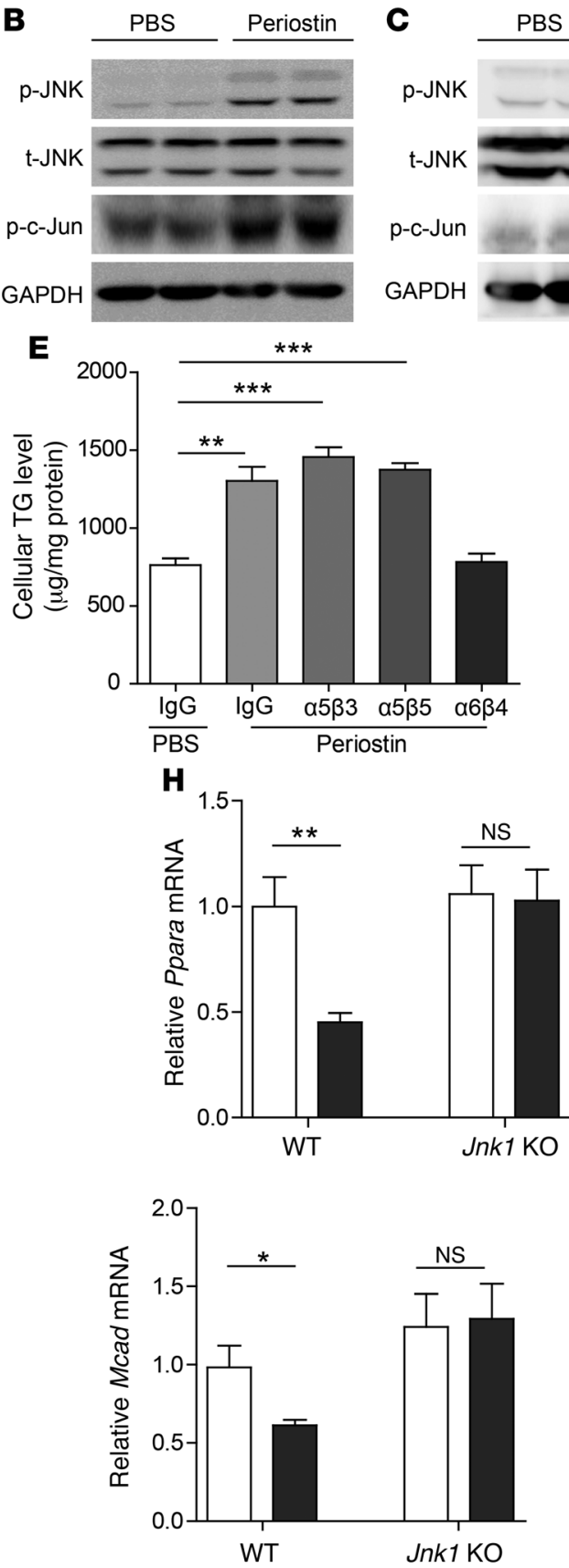
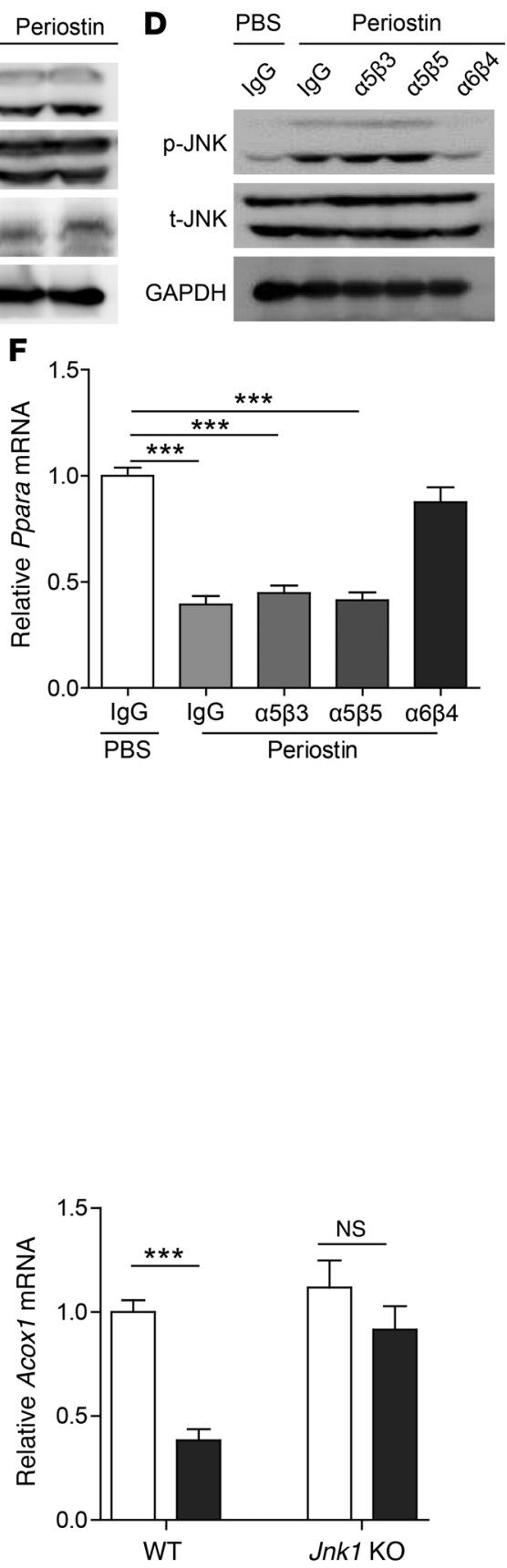

Figure 4. Periostin activates the JNK signaling pathway to promote hepatosteatosis. (A) Phosphorylated and total JNK, c-Jun, p38, AKT, and ERK in the liver of C57BL/6 mice injected with GFP or Postn adenovirus. Total JNK, c-Jun, p38, AKT, and ERK were used as loading controls. (B and C) Phosphorylated and total JNK and c-Jun in HepG2 cells (B) and MPHs (C) incubated with PBS or periostin protein for 1 hour. (D) Phosphorylated and total JNK in MPHs incubated with PBS or periostin protein. Cells were preincubated with Abs against the indicated integrins or IgC as a control for 2 hours, and then treated with PBS or periostin protein for another 1 hour. (E) Cellular TG content in MPHs incubated with PBS or periostin protein. Cells were preincubated with Abs against integrins or IgG control for 2 hours, then treated with PBS or periostin protein for another 36 hours. (F) Ppara mRNA levels in MPHs incubated with PBS or periostin protein. Cells were preincubated with Abs against integrins or lgG control for 2 hours, then treated with PBS or periostin protein for another 24 hours. (C) Cellular TC content in MPHs isolated from WT and $/ \mathrm{nk} 1 \mathrm{KO}$ mice. Cells were treated with periostin protein (50 ng/ml) or PBS for 36 hours $(n=4)$. (H and $\mathbf{I})$ mRNA levels of Ppara $(\mathbf{H})$ and its target genes $(\mathbf{I})$ in MPHs. ${ }^{*} P<0.05,{ }^{* *} P<0.01,{ }^{* *} P<0.001$.

The marked activation of c-Jun seen upon periostin treatment led us to investigate whether c-Jun played a role in the inhibition of Ppara gene transcription. In transient transfection assays using the Ppara-luciferase reporter (-261 Luc) in HepG2 cells, overexpression of c-Jun markedly suppressed the Ppara reporter activity induced by ROR $\alpha$, in the absence and presence of CS (Figure 5C). How- ever, mutations in the ROR $\alpha$ binding site significantly prevented the c-Jun suppression of the Ppara reporter (Figure 5C), which suggests that c-Jun could prevent the ROR $\alpha$ activation of PPAR $\alpha$ and result in inhibition of Ppara gene transcription. Since c-Jun inhibited RORa-induced Ppara gene transcription, we postulated that these 2 molecules might interact with each other. Indeed, co-IP assays in 
A
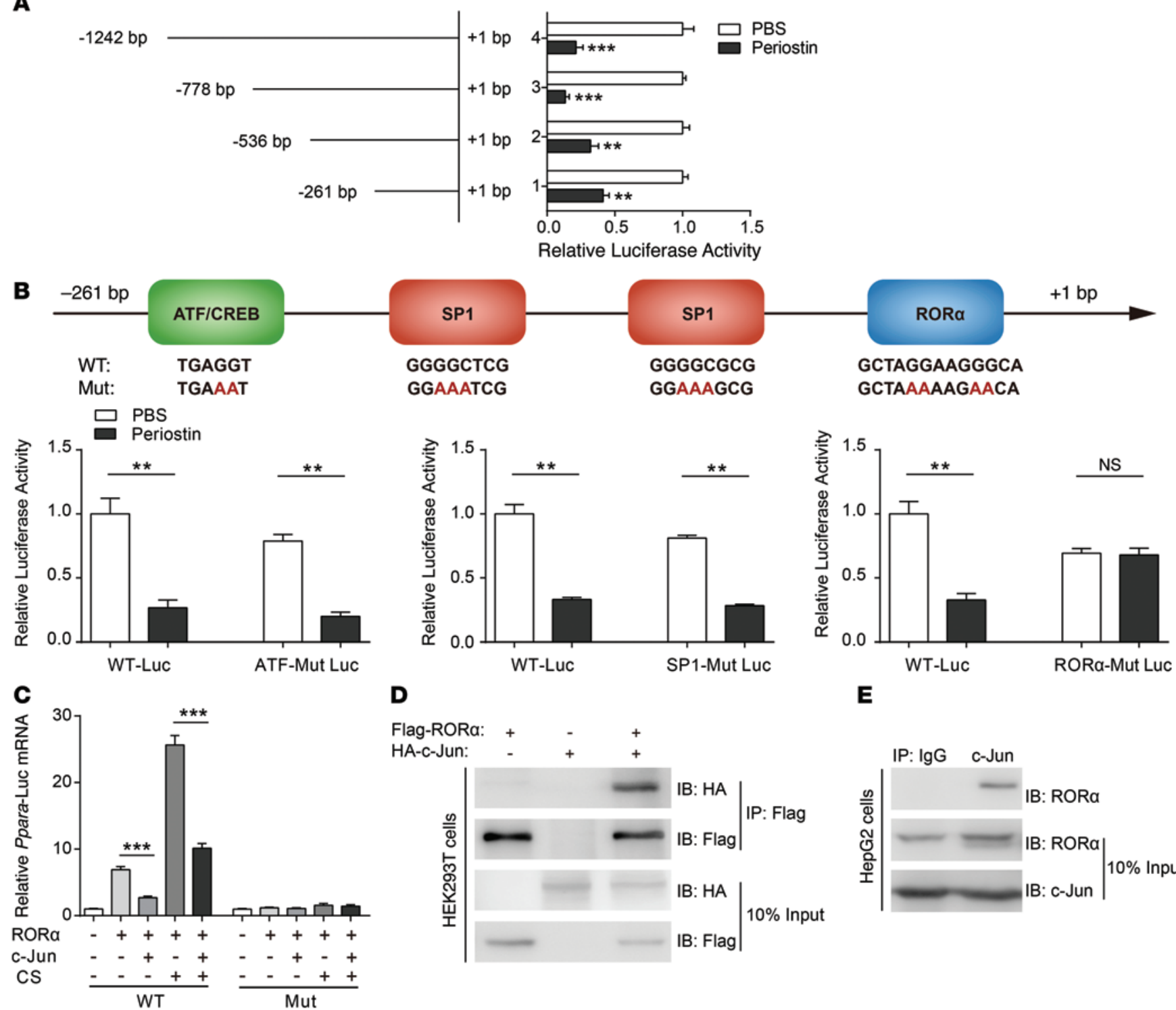

D

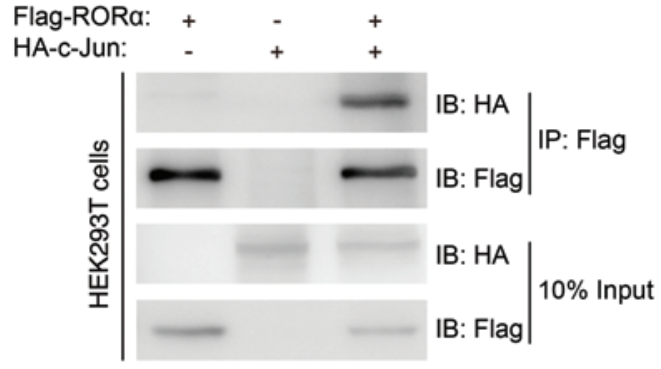

E

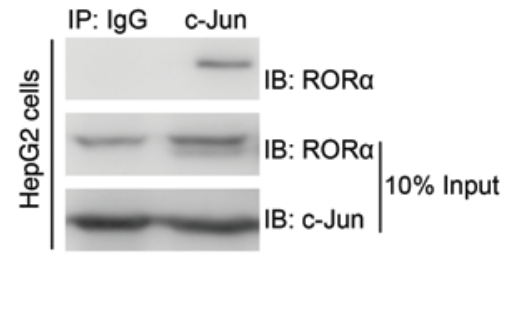

$\mathbf{F}$

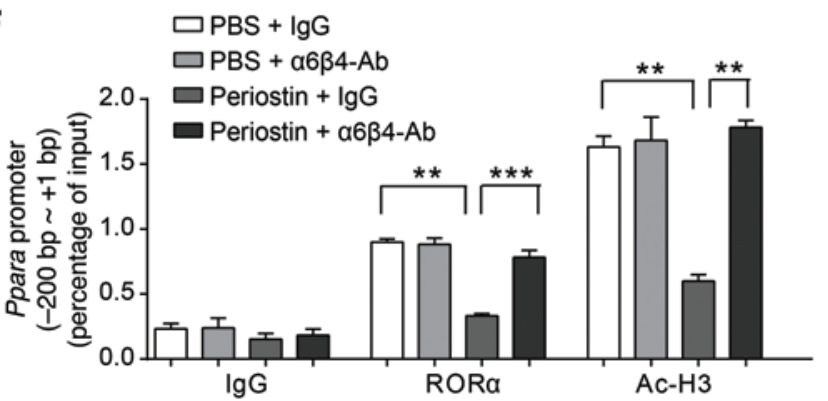

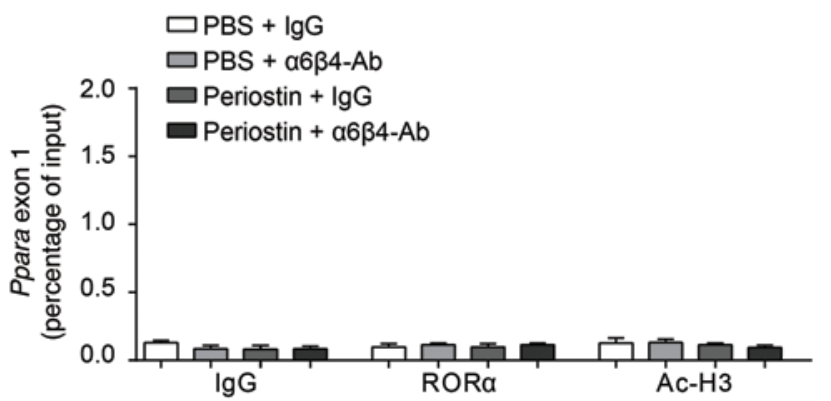

Figure 5. Periostin inhibits PPAR $\alpha$ expression through c-Jun-mediated suppression of ROR $\alpha$ transcriptional activity. (A) Luciferase reporters carrying a series of truncated mouse Ppara promoter. The promoter region from $-1,242$ to +1 bp was cloned and transfected into HepG2 cells. The transcription start site was set as +1 bp. (B) WT and mutant (Mut) Ppara promoters ( -216 to +1 bp) were transfected into HepG2 cells, and luciferase reporter assays were measured. Potential binding sites for ATF/CREB, SP1, and ROR $\alpha$ were mutated as indicated. (C) WT and ROR $\alpha$ binding site mutant Ppara promoters $(-216$ to +1 bp) were cotransfected with ROR $\alpha$, c-Jun, or empty vectors into HepG2 cells. Cells were treated with CS (5 $\mu$ M) or DMSO vehicle control for 12 hours before harvest. (D) ROR $\alpha$ interacted with c-Jun. c-Jun was pulled down by ROR $\alpha$ by IP in HEK293T cells transfected with Flag-tagged ROR $\alpha$ and HA-tagged c-Jun. (E) Interaction of endogenous ROR $\alpha$ and c-Jun. Cell lysates were extracted from HepG2 cells and subjected to IP using c-Jun or IgG Ab and IB using ROR $\alpha \mathrm{Ab}$. (F) ChIP analysis showing binding of ROR $\alpha$ and acetylated histone $\mathrm{H} 3(\mathrm{Ac}-\mathrm{H} 3)$ to the Ppara promoter. MPHs were preincubated with IgG or Abs targeting $\alpha 6 \beta 4$ integrins for 2 hours, then treated with PBS or periostin for another 2 hours. The exon 1 region of $P$ para was used as a negative control. ${ }^{* *} P<0.01,{ }^{* *} P<0.001$. 
A

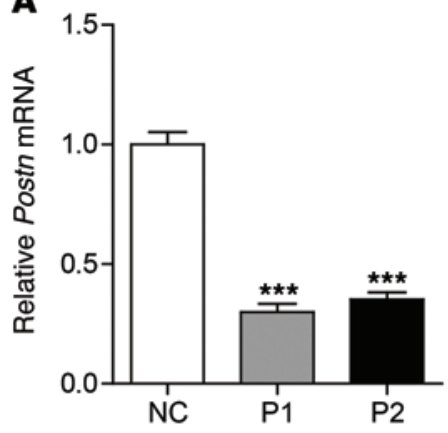

C

NC

P1

P2
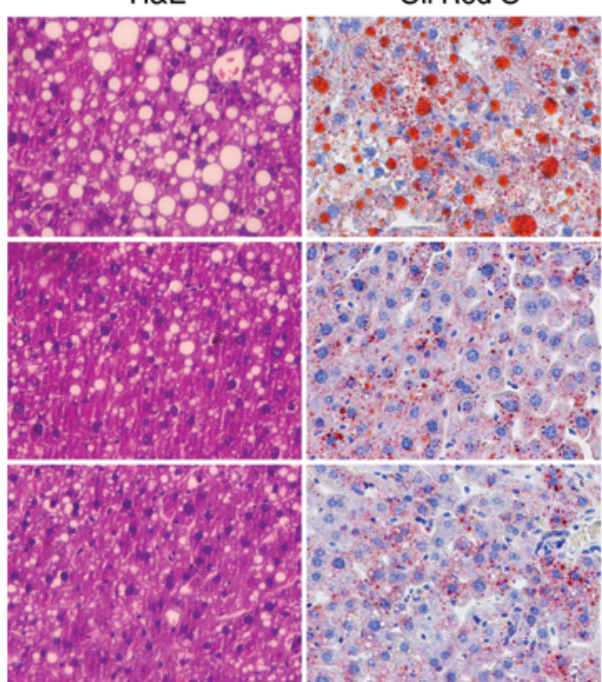

$\mathbf{F}$

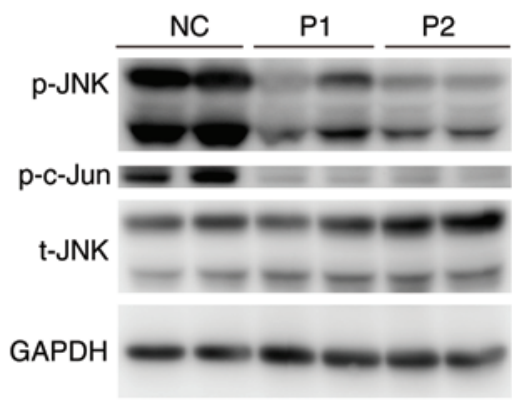

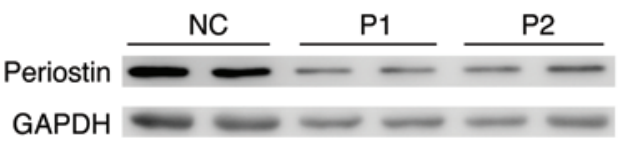

D

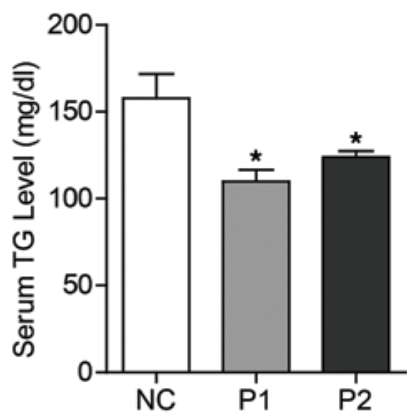

E

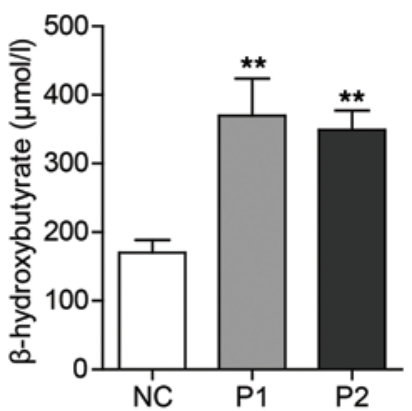

B

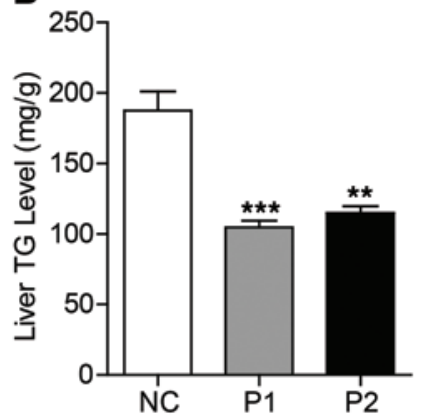

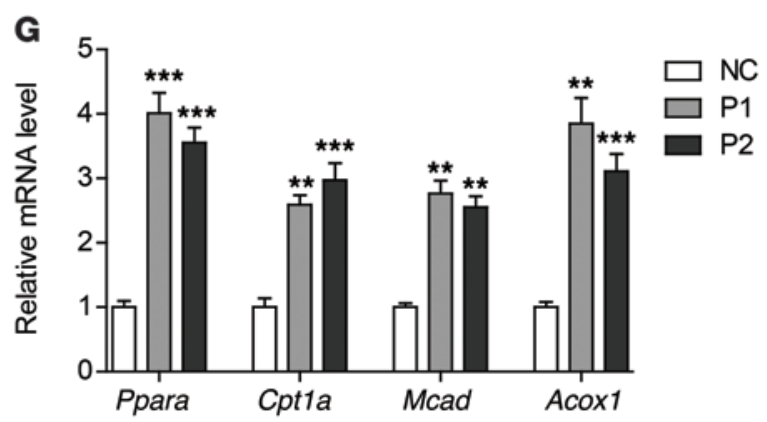

Figure 6. Periostin deficiency improves hepatosteatosis in obese mice. (A) Hepatic Postn mRNA and periostin protein levels in $d b / d b$ mice. Mice were administered with 2 adenoviral shRNAs targeting Postn (P1 and P2) or a negative control (NC) via tail vain injection $(n=5)$. (B) Hepatic TG content in $d b / d b$ mice. (C) Representative hepatic histology by H\&E and Oil Red 0 staining from $d b / d b$ mice. Original magnification, $\times 200$. (D and E) Serum TC (D) and $\beta$-hydroxybutyrate (E) levels in $d b / d b$ mice. (F) Phosphorylated JNK and c-Jun were determined in $d b / d b$ mice with Postn knockdown or controls. (G) mRNA levels of fatty acid oxidation-related genes in mice as in $\mathbf{F}$. ${ }^{*} P<0.05$, ${ }^{* *} P<0.01,{ }^{* *} P<0.001$.

HEK293T and HepG2 cells demonstrated that c-Jun could interact with ROR $\alpha$ (Figure 5, D and E). Furthermore, ChIP assays demonstrated that the amount of ROR $\alpha$ protein bound to the Ppara promoter was significantly reduced in MPHs treated with periostin, which could be prevented by $\alpha 6 \beta 4$ Abs (Figure $5 F$ ). Moreover, acetylated histone $\mathrm{H} 3$, a transcribed gene marker, exhibited changes similar to those observed with ROR $\alpha$ (Figure 5F). Taken together, our data indicate that c-Jun may block ROR $\alpha$ binding to the Ppara promoter, resulting in the inhibition of Ppara gene transcription.

Disruption of Postn improves hepatosteatosis in obese mice. To determine whether knockdown of endogenous Postn improves hepatosteatosis, we disrupted its expression in the liver of $d b / d b$ mice by delivering 2 independent adenoviruses expressing Postnspecific shRNA or adenovirus expressing a nonspecific control shRNA. Postn shRNA treatment significantly reduced hepatic Postn mRNA and periostin protein levels compared with negative control shRNA-injected littermates (Figure 6A). shRNA-mediated loss of hepatic Postn dramatically reduced hepatic TG content, improved hepatosteatosis and serum TG levels, and increased circulating $\beta$-hydroxybutyrate levels (Figure 6, B-E). Fasting glucose levels and insulin sensitivity were improved in $d b / d b$ mice with hepatic Postn knockdown (Supplemental Figure 10, A-C). However, there was no change in BW, fat mass, serum cholesterol levels, liver cholesterol content, alanine aminotransferase (ALT), and as- 

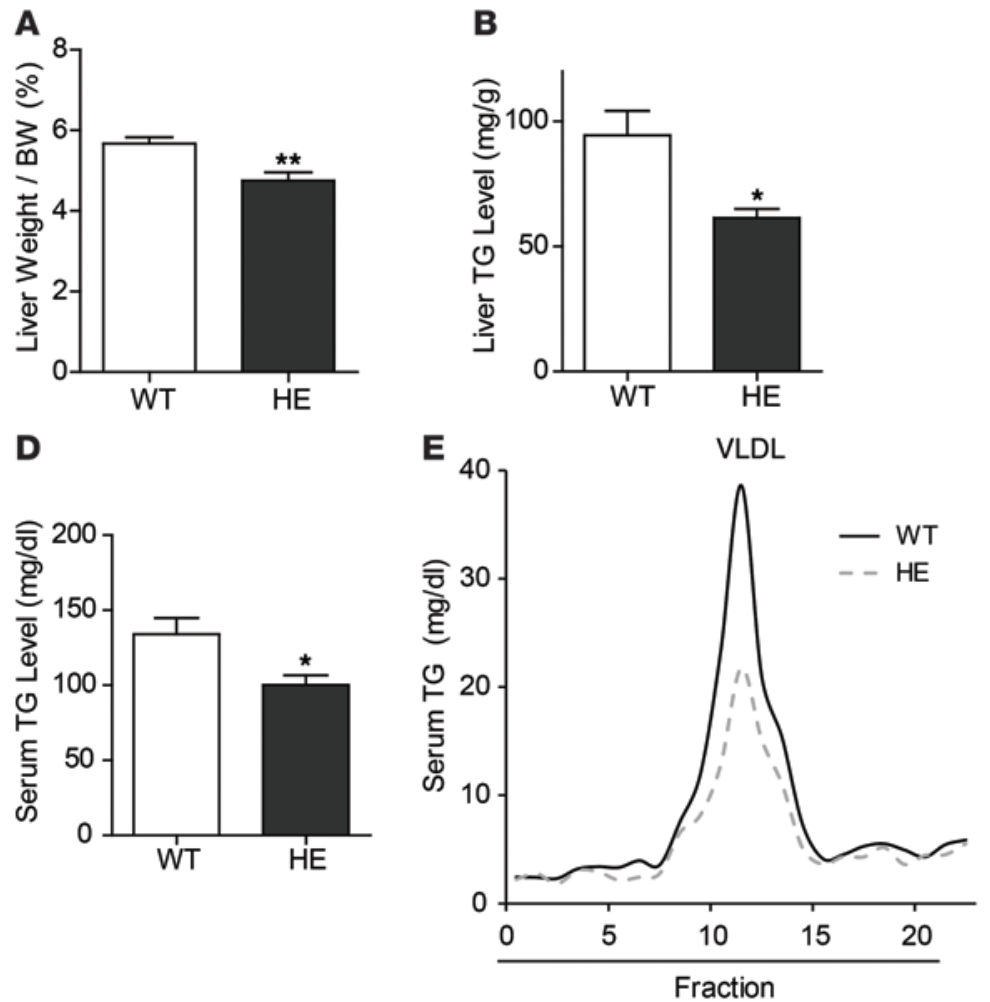

G

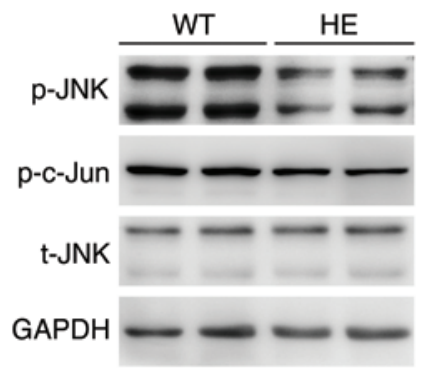

B

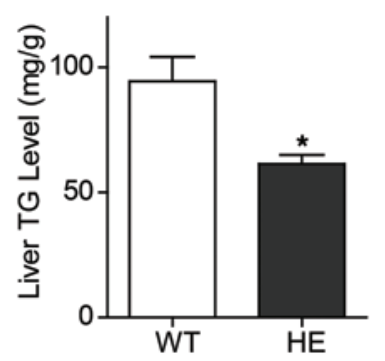

$\mathbf{H}$

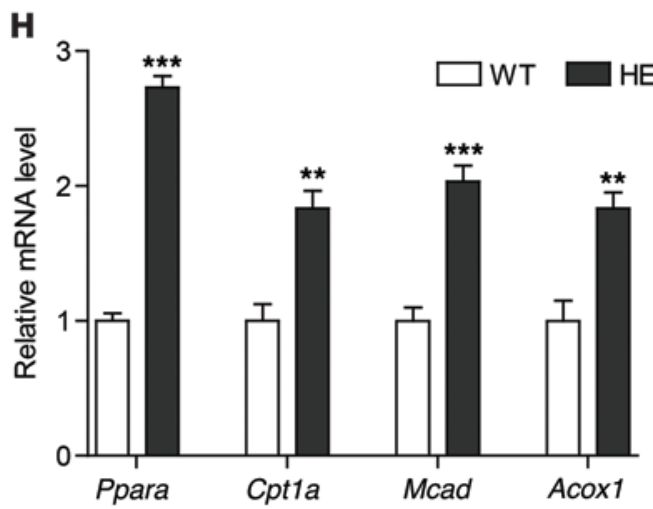

Figure 7. Postn-heterozygous mice show improvement of hepatosteatosis. (A and B) Liver weight (A) and TC content (B) in WT and Postn-heterozygous (HE) mice fed HFD for 8 weeks. $n=5-6$. (C) Representative hepatic histology by H\&E and Oil Red 0 staining from WT and Postn-heterozygous mice. Original magnification, $\times 200$. (D-F) Serum TC (D), lipoprotein-associated TC (E), and $\beta$-hydroxybutyrate (F) levels in WT and Postn-heterozygous mice. (G) Phosphorylated JNK and c-Jun in livers from WT and Postn-heterozygous mice. (H) mRNA levels of Ppara, Cpt1a, Mcad, and Acox1 in livers from WT and Postn-heterozygous mice. ${ }^{*} P<0.05,{ }^{* *} P<0.01,{ }^{* *} P<0.001$.

partate aminotransferase (AST) (Supplemental Figure 11, A-F). At the molecular level, silencing the Postn gene attenuated the activation of JNK pathways and upregulated fatty acid oxidation-related genes (Figure 6, F and G, and Supplemental Figure 11G).

To investigate the long-term effect of periostin deficiency on hepatosteatosis, we used Postn-heterozygous mice, since Postn homozygotes exhibit postnatal growth retardation and skeletal defects $(6,7)$. Postn-heterozygous mice showed a 58\% reduction in Postn mRNA and a $41 \%$ reduction in periostin protein in the liver (Supplemental Figure 12, A and B). WT and Postn-heterozygous mice at the age of 7 weeks were fed HFD for another 8 weeks. HFD caused similar levels of obesity in the 2 groups, as shown by BW, food intake, and fat content (Supplemental Figure 12, C-E). However, liver weight and TG content were significantly reduced in Postn-heterozygous mice
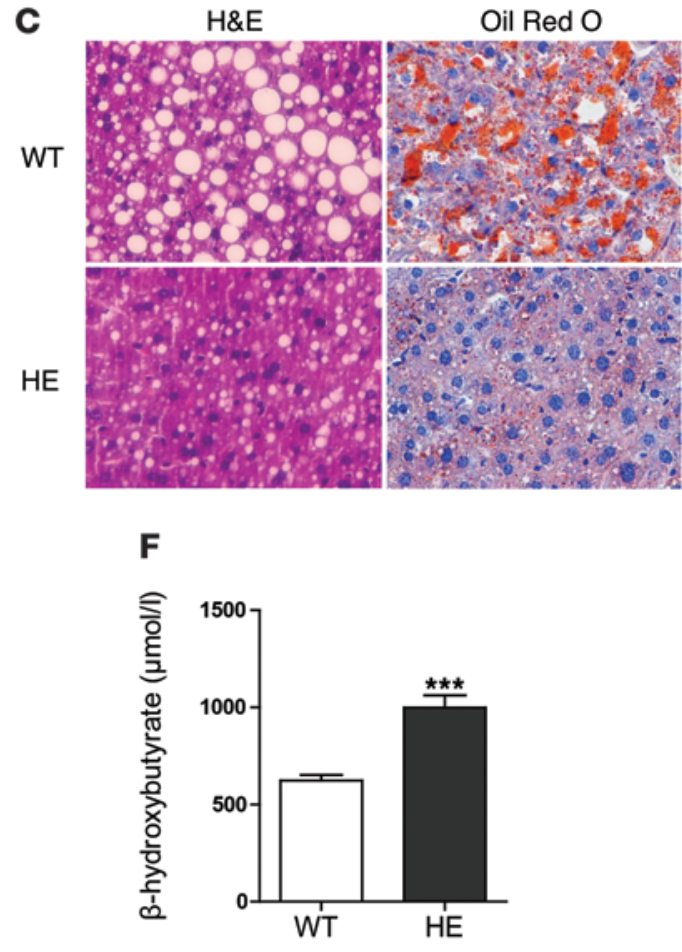

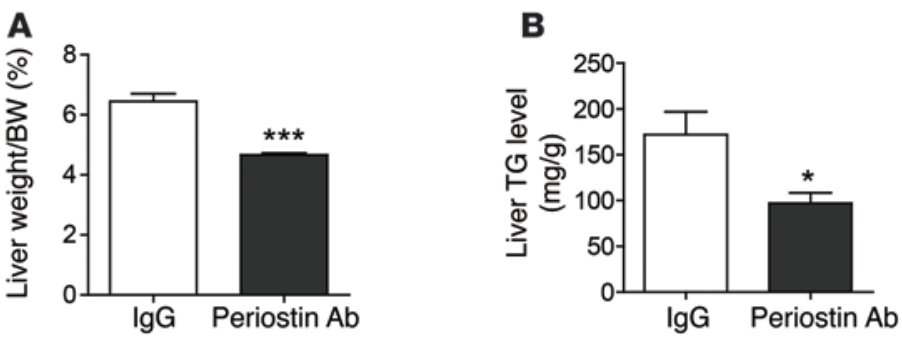

G
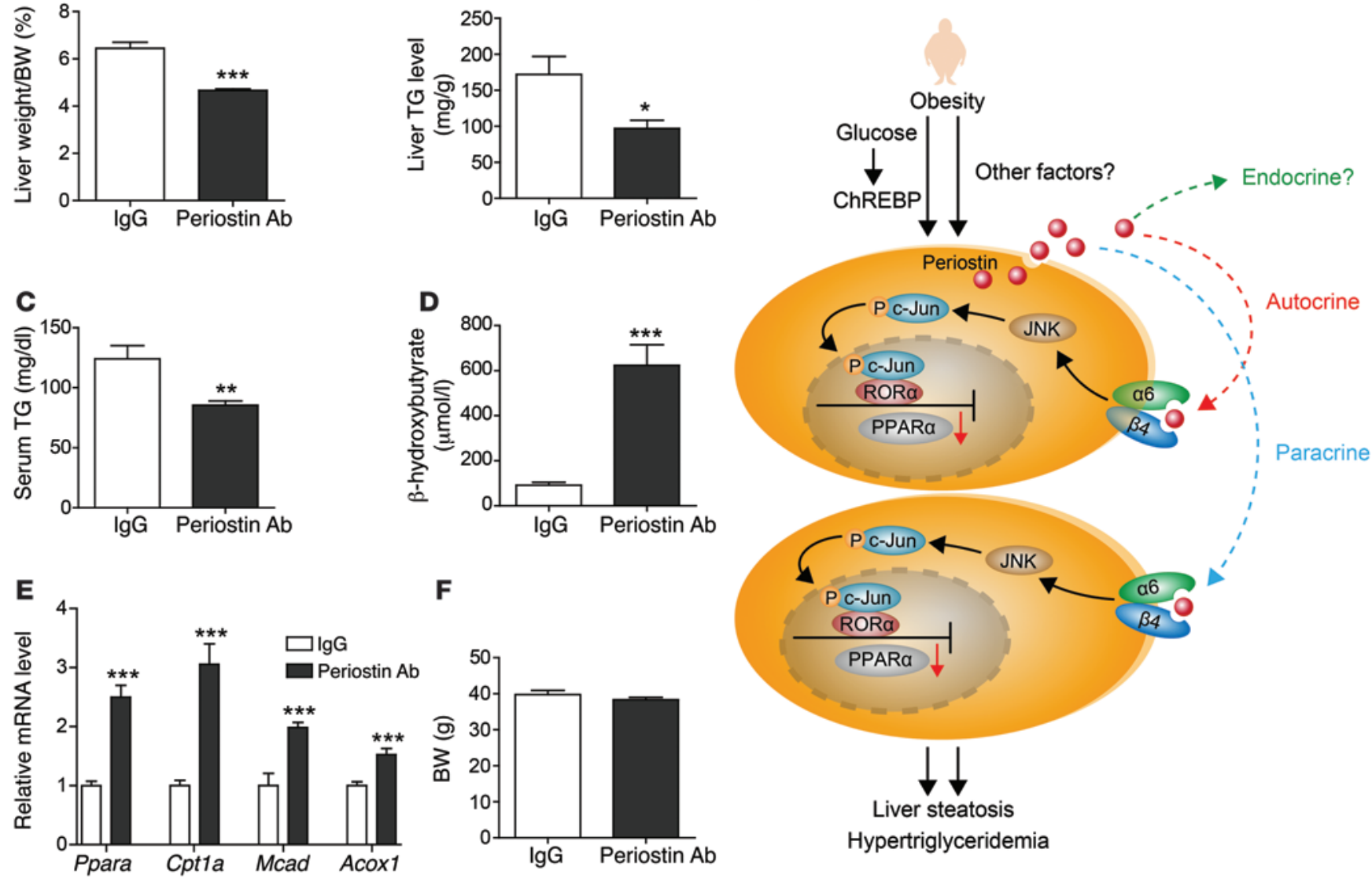

Figure 8. Neutralization of periostin improved hepatosteatosis in $\mathbf{d b} / \mathbf{d} \mathbf{b}$ mice. (A-D) Liver weight (A), hepatic (B) and serum (C) TG content, and $\beta$-hydroxybutyrate level (D) in $d b / d b$ mice treated with control IgG or anti-periostin Ab for 2 weeks. $n=6-8$. (E) qRT-PCR analysis of fatty acid oxidationrelated genes in livers from $d b / d b$ mice treated with control IgG or anti-periostin Ab. (F) BW of $d b / d b$ mice treated with control IgG or anti-periostin Ab. (C) Working model. Periostin is a nutritionally regulated cytokine secreted by the liver. In obese subjects, overproduced periostin binds to $\alpha 6 \beta 4$ integrins, activates the JNK signaling pathway, downregulates PPAR $\alpha$, and consequently leads to hepatosteatosis and hypertriglyceridemia. Our data suggest that periostin may have autocrine or paracrine roles in the liver. Whether hepatic periostin can exert endocrine effects upon distant targets remains to be defined. ${ }^{*} P<0.05,{ }^{* *} P<0.01,{ }^{* *} P<0.001$.

content, and serum TG levels were markedly reduced compared with IgG controls, whereas $\beta$-hydroxybutyrate levels were higher (Figure 8, A-D). Fatty acid oxidation-related genes were also upregulated (Figure $8 \mathrm{E}$ ). Additionally, JNK signaling activation was alleviated, as shown by reduced phosphorylated JNK (Supplemental Figure 13C). However, BW was unchanged (Figure 8F). Taken together, these results indicate that blocking periostin with a neutralizing $\mathrm{Ab}$ can improve hepatosteatosis and hypertriglyceridemia in obese mice.

\section{Discussion}

Our present findings show that aberrant expression of periostin in the liver results in steatosis and hypertriglyceridemia through JNK-mediated suppression of fatty acid oxidation (Figure 8G). Nutrients, including high glucose concentrations, can upregulate periostin expression. Although glucose-induced repression of fatty acid oxidation has been previously studied $(32,33)$, the underlying molecular pathway is poorly understood. Therefore, these results also reveal a novel mechanism clarifying how glucose can regulate hepatic lipid metabolism. We also demonstrated that palmitate could increase periostin expression in HepG2 cells and MPHs (Supplemental Figure 14), which suggests that fatty acids might also be involved in regulation of periostin expression in obesity-associated hepatosteatosis.

It has been reported that periostin exerts its biological functions through several signaling pathways in the development of human cancers, including PI3K/AKT and Wnt/ $\beta$-catenin (12, $34,35)$. However, AKT phosphorylation was not altered in livers of mice overexpressing periostin. Indeed, a large portion of periostin's effects in hepatic lipid management seems to depend on JNK activation, which suggests that this kinase represents a main mechanistic checkpoint for periostin action in the liver. Although this inconsistency in mechanism for cancer and metabolic disorders currently remains unexplored, the downstream signaling activated by periostin might be cell or tissue specific. In addition, studies using genetically engineered mice have shown that hyperactivation of the JNK pathway contributes to the development of metabolic diseases, including hepatic steatosis, obesity, and insulin resistance (36-38). Consistent with this, strong activation of JNK has been observed in the livers of HFD-fed mice and genetically obese mice $(39,40)$. Interestingly, only JNK1 deficiency has been shown to attenuate hepatocyte damage and steatosis, while 
JNK2 deficiency actually increased liver injury (41), showing the different roles of $2 \mathrm{JNKs}$ in hepatic lipid metabolism (42).

Our results indicate that hepatic periostin overproduction phenocopies PPAR $\alpha$ deficiency with respect to TG levels, and loss of PPAR $\alpha$ was sufficient to significantly blunt periostin's effects. It has been well established that disorders of hepatic fatty acid oxidation, including deficiency in PPAR $\alpha$ or its coactivators, lead to massive hepatic TG accumulation and hypertriglyceridemia in animals (43-47). Moreover, some emerging evidence has shown that hepatic fatty acid oxidation is also impaired in human NAFLD. For instance, it was reported that variants in Ppara or Pgcla, a coactivator of PPAR $\alpha$, were significantly associated with NAFLD $(48,49)$. Deficiency of those genes involved in fatty acid oxidation, such as $M C A D$, long-chain acyl-CoA dehydrogenase ( $L C A D)$, and long-chain 3-hydroxyacyl-CoA dehydrogenase ( $L C H A D)$, were also shown to affect the development of human NAFLD $(50,51)$. In addition, some studies revealed that PPAR $\alpha$ expression was downregulated by $50 \%$ in human NAFLD $(52,53)$. Consistently, our data showed that serum $\beta$-hydroxybutyrate levels were significantly reduced in NAFLD patients (Supplemental Figure 15, A-D). Moreover, hepatic TG content was inversely correlated with serum $\beta$-hydroxybutyrate level (Supplemental Figure 15, $\mathrm{E}-\mathrm{G})$. Previous studies have shown that the $\mathrm{L} 162 \mathrm{~V}$ variant of PPARA was significantly associated with several components of the metabolic syndrome, including fasting hypertriglyceridemia (54-56). We also found that obese subjects with the PPARA L162V genotype had a higher hepatic TG content (Supplemental Figure 16). Taken together, these results suggest that PPAR $\alpha$ or fatty acid oxidation disorders may also participate in the development of human NAFLD. In addition, some studies have demonstrated that PPAR $\alpha$ agonist improved liver steatosis in several obese mice models (57-59). However, the effect of PPAR $\alpha$ agonists on improving human NAFLD is still controversial, although some studies have shown positive effects $(60,61)$. Therefore, large, long-term prospective clinical studies are still needed to fully assess the effects of PPAR $\alpha$ agonists.

In agreement with our observations in the liver, suppression of Ppara transcription by JNK has also been shown in the heart (62), although the molecular mechanism remains largely unexplored. We identified a putative binding motif for ROR $\alpha$ in the proximal promoter region of Ppara. It was reported that ROR $\alpha$ regulated lipid homeostasis in skeletal muscle through upregulation of caveolin-3 and CPT-1 $\alpha$ (63). Additionally, activation or overexpression of ROR $\alpha$ attenuated hepatic steatosis through AMPK-mediated inhibition of Lxra transcriptional activity (31). Therefore, our present results reveal an additional mechanism that reinforces the effect of ROR $\alpha$ on hepatic lipid metabolism. The perturbed function of ROR $\alpha$ by JNK/ c-Jun contributed to the periostin-inhibiting effect of Ppara transcription. Co-IP studies in HEK293T and HepG2 cells uncovered an interaction between c-Jun and ROR $\alpha$, by which c-Jun blocked the recruitment of ROR $\alpha$ to the Ppara promoter region (Figure 5, D and E). It has previously been shown that c-Jun participated in active repression of several nuclear transcription factors, such as MyoD, SMAD3, and HNF1 $\alpha$ (64-66). However, further studies are still required to establish the precise mechanisms, which may involve the recruitment of transcriptional corepressors or other alternative linker proteins.

Recent studies have demonstrated that the liver can produce several proteins and secrete them into the circulation, where they participate in the regulation of hepatic and systemic glucose and lipid metabolism. These include fibroblast growth factor 21 (FGF21), $\alpha 2$-HS-glycoprotein (Fetuin-A), and pancreatic-derived factor (PANDER) (67-69). Intriguingly, the dysregulation of those hepatokines is closely associated with hepatosteatosis and dyslipidemia (68-71). Our present results indicate that periostin can also act as a hepatokine to regulate lipid metabolism in the liver. However, whether hepatic periostin has endocrine effects on distant targets, including adipose tissues or skeletal muscles (Figure 8G), awaits further in-depth analysis in future studies.

Together, our findings provided novel insight into the regulation of hepatic TG homeostasis and the mechanism of hepatosteatosis in obese rodents and humans. We identified periostin as an important regulatory molecule in the development of fatty liver. Periostin might therefore be a therapeutic target for fatty liver and dyslipidemia.

\section{Methods}

Further information can be found in Supplemental Methods.

Animal experiments. Male C57BL/6, ob/ob, and $d b / d b$ mice aged 8-12 weeks were purchased from the Shanghai Laboratory Animal Company (SLAC). Post $n$ and Ppara KO mice were obtained from Jackson Laboratories. Jnk1 KO mice were provided by G. Chen (Shanghai Jiao Tong University School of Medicine). All mice were housed at $21^{\circ} \mathrm{C} \pm 1^{\circ} \mathrm{C}$ with humidity of $55 \% \pm 10 \%$ and a 12 -hour light/12-hour dark cycle. HFD-induced obese mice were maintained with free access to high-fat chow (D12492; Research Diets) and drinking water. HFD contained $60 \% \mathrm{kcal}$ from fat, $20 \% \mathrm{kcal}$ from carbohydrate, and $20 \% \mathrm{kcal}$ from protein. ND contained $10 \% \mathrm{kcal}$ from fat, $70 \% \mathrm{kcal}$ from carbohydrate, and $20 \% \mathrm{kcal}$ from protein.

Human subjects. In total, 1,043 adult participants were enrolled in a human cross-sectional study from April to August 2011, as described previously (15). Subjects were screened with physical examination and type B ultrasonography for fatty liver in the Lian Qian community (Xiamen, China). All participants completed a standard questionnaire including physical activity, diet, and history of present and past illnesses and medications. Anthropometric measurements included BW, height, waist circumference, blood pressure (BP), BMI, and body fat. BMI was calculated as weight (in kilograms) divided by the square of the height (in meters). Waist circumference was measured at the midpoint between the inferior costal margin and the superior border of the iliac crest on the midaxillary line. Those who drank 140 or $70 \mathrm{~g} /$ week of alcohol (for men or women, respectively), at the time or in the previous 6 months, were excluded from the study. HBV- or HCV-infected subjects were excluded. For analysis of serum periostin levels, blood samples were collected from 135 NAFLD patients with TG content $>20 \%$ and 105 aged-matched normal subjects with hepatic TG content $<5 \%$, defined using Proton Magnetic Resonance Spectroscopy ( $\left.{ }^{(} \mathrm{H}-\mathrm{MRS}\right)$ (15). For analysis of hepatic periostin concentrations and TG contents, liver biopsy was performed in those subjects who donated their partial livers for liver transplantation. The subjects were screened with physical examination and type B ultrasonography. H\&E staining was performed for histology analysis.

Cell culture. HepG2 cell lines were purchased from the Cell Bank of Type Culture Collection, Chinese Academy of Sciences (CAS), and maintained in DMEM (Gibco) supplemented with 10\% FBS (Gibco), $100 \mathrm{IU} / \mathrm{ml}$ penicillin, and $100 \mu \mathrm{g} / \mathrm{ml}$ streptomycin. MPHs were isolated from adult C57BL/6 mice (8-10 weeks of age) by collagenase perfusion and purified by centrifugation. Freshly prepared hepatocytes were 
seeded at a final density of $0.5 \times 10^{6}$ cells/well in 6-well plates in attachment media (ScienCell). The media were replaced with DMEM (Gibco) at 24 hours. Periostin recombinant protein was purchased from Abcam.

Hepatic and cellular TG measurement. Liver tissues were homogenized in chloroform/methanol (2:1 v/v) using a Polytron tissue grinder (Kinematica AG). Lipid extracts were prepared by the classical Folch method. Extracts were dried under $\mathrm{N}_{2}$ flow and dissolved in isopropanol. For the in vitro model of cellular steatosis, HepG2 cells or MPHs were exposed to periostin protein $(50 \mathrm{ng} / \mathrm{ml}$; Abcam) or PBS vehicle control. TG and cholesterol contents were measured using commercial kits (Biovision) according to the manufacturer's instructions.

Fast protein liquid chromatography. Serum from 5 mice per experimental group was pooled and subjected to fast protein liquid chromatography. VLDL and TG was measured in the eluted fractions using commercial kits (Biovision).

Microarray analysis and $q R T-P C R$. Affymetrix array hybridization and scanning using Mouse Genome 4302.0 chips (Gene Tech Co. Ltd.) has been previously described (13). The full datasets were deposited in GEO (accession no. GSE57425). In order to quantify the transcripts of the genes of interest, qRT-PCR was performed using SYBR Green Premix Ex Taq (Takara) on a Light Cycler 480 (Roche). See Supplemental Methods for primer sequences.

Western blot. Hepatic tissues or cells were lysed in radioimmunoprecipitation buffer containing $50 \mathrm{mM}$ Tris- $\mathrm{HCl}, 150 \mathrm{mM} \mathrm{NaCl}, 5 \mathrm{mM}$ $\mathrm{MgCl}_{2}, 2 \mathrm{mM}$ EDTA, $1 \mathrm{mM} \mathrm{NaF}, 1 \% \mathrm{NP}-40$, and $0.1 \%$ sodium dodecyl sulfate. Western blot was performed using Abs against ChREBP (ab157153; Abcam), periostin (SAB4300696; Sigma-Aldrich), PPAR $\alpha$ (MAB3890; Millipore), JNK (4668, 9258; Cell Signaling), c-Jun (2993, 9165; Cell Signaling), p38 (9215, 2387; Cell Signaling), AKT (13038, 4821; Cell Signaling), ERK (4377, 4695; Cell Signaling), Rac1 (ab33186; Abcam), ROR $\alpha$ (ab60134; Abcam), histone H3 (Ah433; Beyotime), Flag (8146; Cell Signaling), HA (5017; Cell Signaling), and GAPDH (5174; Cell Signaling).
Periostin neutralizing $A b$. The hybridoma cell lines secreting mouse $\mathrm{mAb}$ against mouse periostin were generated by Abmart Co., according to the standard hybridoma technique (72). The mouse mAbs were purified by Protein A affinity chromatograph. mAb purity was confirmed by HPLC. The concentrations of the obtained mAbs were measured by Mouse IgG ELISA Quantitation kit (Bethyl Laboratories), following the manufacturer's instructions. The kinetic parameters of the mAbs were determined using a Biacore T100 instrument (Biacore $A B)$. Purified $A b$ was diluted in saline and injected at a dose of $5 \mathrm{mg} / \mathrm{kg}$ into $d b / d b$ mice for 2 weeks.

Statistics. All values are shown as mean \pm SEM. Statistical differences were determined by 2-way ANOVA with Bonferroni-adjusted post-test or by 2-tailed Student's $t$ test. A $P$ value less than 0.05 was considered significant.

Study approval. The animal protocol was reviewed and approved by the Animal Care Committee of Shanghai Jiao Tong University School of Medicine. The human study was approved by the Human Research Ethics Committee of the Xiamen First Hospital, Xiamen University (Xiamen, China) and the Ethics Committee of Shanghai Jiao Tong University School of Medicine. Written informed consent was obtained from each subject.

\section{Acknowledgments}

This study was supported by grants from National Key Basic Research Program of China (973 Program) (no. 2012CB524902), China Natural Science Foundation (nos. 81321001, 81200636, 81370959, 81300697, 81070681, and 813111105), and Shanghai Committee for Science and Technology (no. 11DZ2270200).

Address correspondence to: Guang Ning and Xiaoying Li, Shanghai Institute of Endocrinology and Metabolism, 197 Ruijin 2nd Road, Shanghai 200025, China. Phone: 86.21.54660108; E-mail: guangning@medmail.com (G. Ning), lixy@sibs.ac.cn (X. Li).
1. Angulo P. Nonalcoholic fatty liver disease. $N$ Engl JMed. 2002;346(16):1221-1231.

2. Cohen JC, Horton JD, Hobbs HH. Human fatty liver disease: old questions and new insights. Science. 2011;332(6037):1519-1523.

3. Hebbard L, George J. Animal models of nonalcoholic fatty liver disease. Nat Rev Gastroenterol Hepatol. 2011;8(1):35-44.

4. Anstee QM, Targher G, Day CP. Progression of NAFLD to diabetes mellitus, cardiovascular disease or cirrhosis. Nat Rev Gastroenterol Hepatol. 2013;10(6):330-344.

5. Fabbrini E, Sullivan S, Klein S. Obesity and nonalcoholic fatty liver disease: biochemical, metabolic, and clinical implications. Hepatology. 2010;51(2):679-689.

6. Rios H, et al. Periostin null mice exhibit dwarfism, incisor enamel defects, and an early-onset periodontal disease-like phenotype. Mol Cell Biol. 2005;25(24):11131-11144.

7. Merle B, Garnero P. The multiple facets of periostin in bone metabolism. Osteoporos Int . 2012;23(4):1199-1212.

8 . Bao S, et al. Periostin potently promotes metastatic growth of colon cancer by augmenting cell survival via the Akt/PKB pathway. Cancer Cell.
2004;5(4):329-339.

9. Kudo Y, et al. Periostin promotes invasion and anchorage-independent growth in the metastatic process of head and neck cancer. Cancer Res. 2006;66(14):6928-6935.

10. Michaylira CZ, et al. Periostin, a cell adhesion molecule, facilitates invasion in the tumor microenvironment and annotates a novel tumorinvasive signature in esophageal cancer. Cancer Res. 2010;70(13):5281-5292.

11. Malanchi I, et al. Interactions between cancer stem cells and their niche govern metastatic colonization. Nature. 2011;481(7379):85-89.

12. Ruan K, Bao S, Ouyang G. The multifaceted role of periostin in tumorigenesis. Cell Mol Life Sci. 2009;66(14):2219-2230.

13. Lu Y, et al. Yin Yang 1 promotes hepatic steatosis through repression of farnesoid $\mathrm{X}$ receptor in obese mice. Gut. 2014;63(1):170-178.

14. Clee SM, Attie AD. The genetic landscape of type 2 diabetes in mice. Endocr Rev. 2007;28(1):48-83.

15. Zhang HJ, et al. Irisin is inversely associated with intrahepatic triglyceride contents in obese adults. J Hepatol. 2013;59(3):557-562.

16. Zhang H, et al. Glucose-mediated repression of menin promotes pancreatic $\beta$-cell proliferation.
Endocrinology. 2012;153(2):602-611.

17. Uyeda K, Repa JJ. Carbohydrate response element binding protein, ChREBP, a transcription factor coupling hepatic glucose utilization and lipid synthesis. Cell Metab. 2006;4(2):107-110.

18. Benhamed F, et al. The lipogenic transcription factor ChREBP dissociates hepatic steatosis from insulin resistance in mice and humans. J Clin Invest. 2012;122(6):2176-2194.

19. Postic C, Girard J. Contribution of de novo fatty acid synthesis to hepatic steatosis and insulin resistance: lessons from genetically engineered mice. J Clin Invest. 2008;118(3):829-838.

20. Reddy JK, Rao MS. Lipid metabolism and liver inflammation. II. Fatty liver disease and fatty acid oxidation. Am J Physiol Gastrointest Liver Physiol. 2006;290(5):852-858.

21. Hiukka A, Maranghi M, Matikainen N, Taskinen MR. PPARalpha: an emerging therapeutic target in diabetic microvascular damage. Nat Rev Endocrinol. 2010;6(8):454-463.

22. Bennett BL, et al. SP600125, an anthrapyrazolone inhibitor of Jun N-terminal kinase. Proc Natl Acad Sci US A. 2001;98(24):13681-13686.

23. Berrier AL, Martinez R, Bokoch GM, LaFlamme $\mathrm{SE}$. The integrin beta tail is required and suffi- 
cient to regulate adhesion signaling to Rac1.JCell Sci. 2002;115(pt 22):4285-4291.

24. Nodari A, et al. $\beta 1$ Integrin activates Rac1 in Schwann cells to generate radial lamellae during axonal sorting and myelination. JCell Biol. 2007;177(6):1063-1075.

25. Kaempchen K, Mielke K, Utermark T, Langmesser S, Hanemann CO. Upregulation of the Rac1/JNK signaling pathway in primary human schwannoma cells. Hum Mol Genet. 2003; 12(11):1211-1221.

26. Sharma M, Urano F, Jaeschke A. Cdc42 and Rac1 are major contributors to the saturated fatty acidstimulated JNK pathway in hepatocytes. J Hepatol. 2012;56(1):192-198.

27. Gao Y, Dickerson JB, Guo F, Zheng J, Zheng Y. Rational design and characterization of a Rac GTPase-specific small molecule inhibitor. Proc Natl Acad Sci USA. 2004;101(20):7618-7623.

28. Pineda Torra I, Jamshidi Y, Flavell DM, Fruchart JC, Staels B. Characterization of the human PPARalpha promoter: identification of a functional nuclear receptor response element. Mol Endocrinol. 2002;16 (5):1013-1028.

29. Ma X, et al. Deletion of steroid receptor coactivator-3 gene ameliorates hepatic steatosis. J Hepatol. 2011;55(2):445-452.

30. Maglich JM, Lobe DC, Moore JT. The nuclear receptor CAR (NR113) regulates serum triglyceride levels under conditions of metabolic stress. JLipid Res. 2009;50(3):439-445.

31. Kim EJ, et al. Retinoic acid receptor-related orphan receptor $\alpha$-induced activation of adenosine monophosphate-activated protein kinase results in attenuation of hepatic steatosis. Hepatology. 2012;55(5):1379-1388.

32. Ravnskjaer K, Boergesen M, Dalgaard LT, Mandrup S. Glucose-induced repression of PPAR $\alpha$ gene expression in pancreatic beta-cells involves PP2A activation and AMPK inactivation. J Mol Endocrinol. 2006;36(2):289-299.

33. Boergesen M, Poulsen Ll, Schmidt SF, Frigerio F, Maechler P, Mandrup S. ChREBP mediates glucose repression of peroxisome proliferator -activated receptor $\alpha$ expression in pancreatic $\beta$-cells. J Biol Chem. 2011;286(15):13214-13225.

34. Utispan K. Periostin activates integrin $\alpha 5 \beta 1$ through a PI3K/AKT -dependent pathway in invasion of cholangiocarcinoma. Int JOncol. 2012;41(3):1110-1118.

35. Ouyang G, Liu M, Ruan K, Song G, Mao Y, Bao S. Upregulated expression of periostin by hypoxia in non-small-cell lung cancer cells promotes cell survival via the Akt/PKB pathway. Cancer Lett. 2009;281(2):213-219.

36. Hirosumi J, et al. A central role for JNK in obesity and insulin resistance. Nature. 2002; 420(6913):333-336.

37. Czaja MJ. JNK regulation of hepatic manifestations of the metabolic syndrome. Trends Endocrinol Metab. 2010;21(12):707-713.

38. Seki E, Brenner DA, Karin M. A liver full of JNK: signaling in regulation of cell function and disease pathogenesis, and clinical approaches. Gastroenterology. 2012;143(2):307-320.

39. Solinas G, Naugler W, Galimi F, Lee MS, Karin M. Saturated fatty acids inhibit induction of insulin gene transcription by JNK-mediated phosphory- lation of insulin-receptor substrates. Proc Natl Acad Sci U S A. 2006;103(44):16454-16459.

40. Tuncman G, Hirosumi J, Solinas G, Chang L, Karin M, Hotamisligil GS. Functional in vivo interactions between JNK1 and JNK2 isoforms in obesity and insulin resistance. Proc Natl Acad Sci U S A. 2006;103(28):10741-10746.

41. Schattenberg JM, et al. JNK1 but not JNK2 promotes the development of steatohepatitis in mice. Hepatology. 2006;43(1):163-172.

42. Singh R, Wang Y, Xiang Y, Tanaka KE, Gaarde WA, Czaja MJ. Differential effects of JNK1 and JNK2 inhibition on murine steatohepatitis and insulin resistance. Hepatology. 2009;49(1):87-96.

43. Lefebvre P, Chinetti G, Fruchart JC, Staels B. Sorting out the roles of PPAR $\alpha$ in energy metabolism and vascular homeostasis. JClin Invest. 2006;116(3):571-580.

44. Kersten S, Seydoux J, Peters JM, Gonzalez FJ, Desvergne B, Wahli W. Peroxisome proliferatoractivated receptor $\alpha$ mediates the adaptive response to fasting. JClin Invest. 1999; 103(11):1489-1498.

45. Li S, et al. Genome-wide coactivation analysis of PGC-1 $1 \alpha$ identifies BAF60a as a regulator of hepatic lipid metabolism. Cell Metab. 2008; 8(2):105-117.

46. Purushotham A, Schug TT, Xu Q, Surapureddi S, Guo X, Li X. Hepatocyte-specific deletion of SIRT1 alters fatty acid metabolism and results in hepatic steatosis and inflammation. Cell Metab. 2009;9(4):327-338.

47. Kulozik P, et al. Hepatic deficiency in transcriptional cofactor TBL1 promotes liver steatosis and hypertriglyceridemia. Cell Metab. 2011;13(4):389-400.

48. Chen S, Li Y, Li S, Yu C. A Val227Ala substitution in the peroxisome proliferator activated receptor $\alpha$ (PPAR $\alpha$ ) gene associated with non-alcoholic fatty liver disease and decreased waist circumference and waist-to-hip ratio. J Gastroenterol Hepatol. 2008;23(9):1415-1418.

49. Yoneda M, et al. Association between PPARGC1A polymorphisms and the occurrence of nonalcoholic fatty liver disease (NAFLD). BMC Gastroenterol. 2008;8:27.

50. Saudubray JM, et al. Recognition and management of fatty acid oxidation defects: a series of 107 patients. JInherit Metab Dis. 1999;22(4):488-502.

51. Hooper AJ, Adams LA, Burnett JR. Genetic determinants of hepatic steatosis in man. J Lipid Res. 2011;52(4):593-617.

52. Kohjima M, et al. Re-evaluation of fatty acid metabolism-related gene expression in nonalcoholic fatty liver disease. Int J Mol Med. 2007;20(3):351-358.

53. Videla LA, Pettinelli P. Misregulation of PPAR functioning and its pathogenic consequences associated with nonalcoholic fatty liver disease in human obesity. PPAR Res. 2012;2012:107434.

54. Robitaille J, et al. Association between the PPAR $\alpha$-L162V polymorphism and components of the metabolic syndrome. JHum Genet. 2004;49(9):482-489.

55. Flavell DM, et al. Peroxisome proliferator-activated receptor alpha gene variation influences age of onset and progression of type 2 diabetes. Diabetes. 2005;54(2):582-586.
56. Sparsø T, et al. Relationships between the functional PPARalpha Leu162Val polymorphism and obesity, type 2 diabetes, dyslipidaemia, and related quantitative traits in studies of 5799 middleaged white people. Mol Genet Metab. 2007; 90(2):205-209.

57. Bijland $S$, et al. Fenofibrate increases very low density lipoprotein triglyceride production despite reducing plasma triglyceride levels in APOE*3-Leiden.CETP mice. J Biol Chem. 2010;285(33):25168-25175.

58. Chan SM, et al. Activation of PPAR $\alpha$ ameliorates hepatic insulin resistance and steatosis in high fructose-fed mice despite increased endoplasmic reticulum stress. Diabetes. 2013;62(6):2095-2105.

59. Kostapanos MS, Kei A, Elisaf MS. Current role of fenofibrate in the prevention and management of non-alcoholic fatty liver disease. World J Hepatol. 2013;5(9):470-478.

60. Saibara T, Onishi S, Ogawa Y, Yoshida S, Enzan H. Bezafibrate for tamoxifen-induced non-alcoholic steatohepatitis. Lancet. 1999;353(9166):1802.

61. Athyros VG, et al. Effect of multifactorial treatment on non-alcoholic fatty liver disease in metabolic syndrome: a randomised study. Curr Med Res Opin. 2006;22(5): 873-883.

62. Drosatos K, et al. Inhibition of c-Jun-N-terminal kinase increases cardiac peroxisome proliferator- activated receptor $\alpha$ expression and fatty acid oxidation and prevents lipopolysaccharide-induced heart dysfunction. J Biol Chem. 2011;286(42):36331-36339.

63. Lau P, Nixon SJ, Parton RG, Muscat GE. RORalpha regulates the expression of genes involved in lipid homeostasis in skeletal muscle cells: caveolin-3 and CPT-1 are direct targets of ROR.J Biol Chem. 2004;279(35):36828-36840.

64. Bengal E, et al. Functional antagonism between c-Jun and MyoD proteins: a direct physical association. Cell. 1992;68(3):507-519.

65. Zhang Y, Feng XH, Derynck R. Smad3 and Smad 4 cooperate with c-Jun/c-Fos to mediate TGF-beta-induced transcription. Nature. 1998;394(6696):909-913.

66. Xiong X, et al. Hepatic steatosis exacerbated by endoplasmic reticulum stress-mediated downregulation of FXR in aging mice. J Hepatol. 2014;60(4):847-854.

67. Badman MK, Pissios P, Kennedy AR, Koukos G, Flier JS, Maratos-Flier E. Hepatic fibroblast growth factor 21 is regulated by PPAR $\alpha$ and is a key mediator of hepatic lipid metabolism in ketotic states. Cell Metab. 2007;5(6):426-437.

68. Reinehr T, Roth CL. Fetuin-A and its relation to metabolic syndrome and fatty liver disease in obese children before and after weight loss. J Clin Endocrinol Metab. 2008;93(11):4479-4485.

69. Li J, et al. Pancreatic-derived factor promotes lipogenesis in the mouse liver: role of the Forkhead box 1 signaling pathway. Hepatology. 2011;53(6):1906-1916.

70. Dushay J, et al. Increased fibroblast growth factor 21 in obesity and nonalcoholic fatty liver disease. Gastroenterology. 2010;139(2):456-463.

71. Stefan N, Häring HU. The role of hepatokines in metabolism. Nat Rev Endocrinol. 2013;9(3):144-152.

72. Yokoyama WM, et al. Production of monoclonal antibodies. Curr Protoc Immunol. 2013;102:Unit 2.5. 